\title{
On pricing of perishable assets with menu costs
}

\author{
Emre Berk $^{\mathrm{a}, *}$, Ülkü Gürler ${ }^{\mathrm{a}}$, Gonca Yıldırım ${ }^{\mathrm{b}}$ \\ a Bilkent University, Ankara 06800, Turkey \\ ${ }^{\mathrm{b}}$ University of Florida, Gainesville, FL, USA
}

\section{A R T I C L E I N F O}

\section{Article history:}

Received 27 December 2007

Accepted 12 February 2009

Available online 18 March 2009

Keywords:

Perishable assets

Menu costs

Dynamic pricing

Revenue management

\begin{abstract}
A B S T R A T
We consider dynamic pricing of perishable assets in the presence of price-sensitive renewal demand processes. Unlike the existing works in the literature, we explicitly incorporate non-negligible price change costs which reflects the revenue management practice more realistically. These costs are also known as menu costs in the economic literature. The objective is to maximize the discounted expected profit for an initial inventory of $Q$ items by determining the selling prices dynamically. We employ a dynamic programming approach and formulate a model that captures the pricedemand relationship. We establish some theoretical results on the properties of the problem at hand. Specifically, we establish the sufficient conditions under which the within-period profit is concave in the selling price and in the remaining shelf life and, furthermore, show the structure of the myopically and asymptotically optimal pricing policy. In a numerical study, we investigate the impact of various system parameters and, in particular, the existence of menu costs, on pricing decisions. We observe that ignoring menu costs may be significantly misleading for the implementation of revenue management. We also propose four implementable policy heuristics and examine their performances. Our findings support some results previously obtained in settings with continuous pricing and negligible price change costs; and, contradict some others.
\end{abstract}

(c) 2009 Published by Elsevier B.V.

\section{Introduction}

Successful management of assets within a supply chain entails two fundamental decisions: determination of stocking levels to satisfy given demand levels and determination of price levels to achieve desired demand levels. The first problem is a passive response optimizing within the system whereas the second problem actively manages the operating environment. In this paper, we focus on the latter and revisit the dynamic pricing problem for perishable assets in a stylistic fashion to address some issues observed at a retailer.

Perishable assets can be an inventory of items which have a constant usable shelf life, or a set of airline seats for

\footnotetext{
* Corresponding author.

E-mail address: eberk@bilkent.edu.tr (E. Berk).
}

a flight of a particular date, or a number of hotel rooms to be sold for a Christmas vacation. The dynamic pricing problem associated with such assets is the determination of selling prices to optimize a monetary objective such as maximizing expected profit or maximizing expected revenue. Our work has been motivated by the practices of a retailer whose operational setting exhibit certain features not fully addressed previously in the revenue management literature. First, the retailer faces random demand within the selling season which is typically of unit size per customer arrival, but the inter-arrival time of customers is non-Markovian. Second, price changes are possible but costly within the selling season. Third, the retailer has to jointly determine the initial stocking levels and the pricing decisions within the season.

Non-Markovian demand has received almost no attention in the revenue management literature despite its commonplace and importance in practice. The Markovian 
assumption holds for demand processes with relatively low coefficients of variation. However, in cases where high demand variances are observed (as in high fashion goods or automobiles), non-exponential inter-demand arrival time distributions are more appropriate. The main difficulty with non-Markovian demand in revenue management appears to be modeling the price-sensitivity of the demand process. On one hand, it is not easy to model the demand process parameters as functions of price and, on the other, it is not clear how one can adequately modify the hazard rates if price changes are allowed between demand arrivals.

Price change costs have largely been assumed negligible in the revenue management literature. This may be appropriate in certain settings, especially when the seller takes a passive role in stimulating the demand. However, when a seller takes an active role in promoting the demand via advertisement and/or announcement of new prices, the costs involved may not be negligible despite advances in technology. Such costs associated with price changes are also known in the economics literature as "menu costs" with reference to the particular physical costs of printing new menus at a restaurant or changing labels at a store every time the prices are changed. The existence of positive price change costs is known to create stickiness in the prices and have direct effects on economic output and welfare. (See Blanchard and Fischer (1989) for a discussion of menu costs and their implications for macroeconomic decisions.) Despite their commonness and importance, the impact of menu costs has not been explicitly investigated previously. It may be intuitive that positive price change costs would result in lower expected profits, but how they affect individual pricing decisions over a particular demand realization path is not clear a priori. Furthermore, it is interesting to see how frequently price changes occur in an optimal setting and the impact of change costs on this frequency.

In this paper, we revisit the dynamic pricing problem for perishable assets in a stylistic fashion to address the above issues. Typically, the dynamic pricing problem has focused on merely the determination of the prices over time but the determination of the size of assets (or, initial inventory of items) is considered outside the scope of the pricing decision. However, as we discuss below, some works have considered such joint decisions as well; in our numerical work, we also optimize jointly the initial stock and the pricing decisions for certain settings.

The earliest work known to us on pricing a perishable item with a fixed shelf life is Eilon and Mallya (1966). Cohen (1977) considered joint pricing and ordering policies in a special model with exponential decay and deterministic demand rate. Later, Kang and Kim (1983) and Aggarwal and Jaggi (1989) reformulated and extended this model.

Gallego and van Ryzin (1994) provided one of the more recent and seminal studies on revenue management with multiple prices and unlimited price changes for Poisson demand processes. In their work, the optimal pricing policy was obtained in closed form for Poisson demand processes. For general demand functions, they analyzed a deterministic version of the problem and obtained an upper bound on the revenue. With this upper bound, they were able to develop a single price policy that is asymptotically optimal when either remaining shelf life or inventory volume is large. However, these approximations were criticized by Feng and Xiao (2000a) stating that a large sales volume and a long remaining lifetime usually smooth out the fluctuations in sales over the season. They suggested that this situation is less likely when the remaining time interval and remaining inventory become small. They also implied that only a particular family of demand functions (exponential) was investigated and the results were not tested for small time intervals. For general demand functions, Feng and Gallego (1995) obtained the optimal revenue maximizing policy with two prices and a single switch in a finite horizon yield management setting. They dealt with the optimal timing of the single price change from a given initial price. Feng and Xiao (1999) incorporated a risk factor into the twoprice model. Feng and Gallego (1996) extended the model by assuming time-dependent or Markovian demand and fares. Feng and Xiao (2000b) modified Feng and Gallego (1995)'s model for airline fares setting considering two prices and a single switch assuming predetermined prices and price sensitive demand following Poisson process. In all of the above models, price reversal was not allowed and pricing was either of markup or markdown form only. Feng and Xiao (2000a) extended the work of Gallego and van Ryzin (1994), by assuming one price to be offered at a time among a list of predetermined values and with reversible change in prices. Demand was taken as Poisson process and strictly decreasing in price. The optimal prices maximizing the revenues were computed based on the length of remaining sales time and inventory.

Subrahmanyan and Shoemaker (1996) developed a dynamic programming (DP) model for a periodic review inventory system with uncertain demand and solved it numerically using backward recursion. They incorporated learning and updating of demand by observing the system through previous periods and creating posterior demand distribution via Bayes Rule. They discounted the maximum expected profit so as to find the stocking, reordering quantities and pricing for items with a short sales season such as fashion goods. Federgruen and Heching (1999) analyzed a similar system for periodic review model in which stockouts were fully backlogged. Rajan et al. (1992) analyzed the dynamic pricing and ordering decisions for a monopolistic retailer with continuous deterioration. The perishing was formulated using a time dependent wastage rate and value drop. They investigated linear and nonlinear demand cases and established propositions on the optimal price changes and optimal cycle length. They assumed that the seller knows the parameters of the demand distributions with certainty and no learning or revision of the demand distributions takes place during the horizon. They also compared dynamic pricing with fixed price policies and reported that the difference between profits depends on the extent optimal dynamic prices vary over the cycle. Zhao and Zheng (2000) generalized the basic model of Gallego and van Ryzin (1994) to include the customer reservations price distributions. They obtained the structural properties of the 
optimal policies and established conditions on the inventory- and time-monotonicity of the optimal prices. For settings with multiple products or firms, we can cite the recent works by Maglaras and Meissner (2006) who considered dynamic pricing and capacity allocation strategies and by Diai et al. (2005) who studied pricing decisions for competing actors in a theoretic approach. For a broader review of the theory and practice of revenue management, we refer the reader to Talluri and van Ryzin (2004).

In all of the works cited, price change costs were assumed to be negligible. To the best of our knowledge, Netessine (2006) is the only work where a restricted number of price changes was considered. This restriction alludes to a positive change cost but such costs were not explicitly modeled. Moreover, Netessine (2006) assumed a deterministic demand environment. Hence, we are unaware of any works that consider explicitly positive menu costs in the presence of random demand arrivals. In our work, we attempt to fill this gap.

Our work contributes to revenue management in a number of ways. The first novelty in our work is that we consider non-negligible price change costs and include them directly in our model in astochastic setting. The second fundamental difference between our work and the existing models is the nature of the demand process. We allow for the demand process to be described by renewal processes. As such, our models are applicable to not only Poisson demand but also to non-Markovian demand processes. This generalization enables one to model demand behaviors in settings where demand distributions per time period may be dependent. Thirdly, we make pricing decisions at demand arrival epochs. This, as we shall see below, significantly changes the properties of the revenue management model. Finally, we consider joint optimization of initial stock and dynamic pricing as modeled herein. The methodology we employ is DP. However, we formulate our DP models over periods with random durations, where demand occurrences constitute pricing decision epochs. Based on our model, analytically, we establish the conditions for the within-period expected profit to be concave in selling price, provide the myopically and asymptotically optimal policy structures, and show that the within-period and optimal expected profit functions are concave in the remaining shelf life under certain conditions. We also propose four implementable heuristics and examine their performances. In a numerical study, we (i) supplement our theoretical findings with illustrative examples, and present results on (ii) the impact of the fixed price change cost and various system parameters on the pricing and expected profit profiles over the horizon, (iii) the sensitivity of the optimal starting price and initial stock to various system parameters, and (iv) the performance comparisons of the heuristics.

The rest of the paper is organized as follows. In Section 2 , the basic assumptions are presented and the problem is formulated. Section 3 provides some theoretical results. In Section 4, we introduce the heuristics and present the results of our numerical study. We conclude with a short summary and some possible future work.

\section{Basic assumptions and the model}

We consider a perishable asset inventory of $Q$ items. The items are withdrawn from stock either by unit demands or perishing and there is no replenishment opportunity during the horizon of the problem. All items in stock have the same usable shelf life $\tau$, which also constitutes the horizon of the pricing problem. The initial ordering and purchasing costs are considered sunk. Without loss of generality, we assume that the items left over (unsold) at the end of the horizon have zero salvage value and incur a unit perishing cost of $\pi$, which may include disposal costs. Each unit in stock held per unit time incurs a holding cost of $h$ and each unit sold brings in a revenue equal to the selling price. All cash flows are continuously discounted at a constant rate $r$. Therefore, the discounted revenue for an item sold at price $p$ after being held $x$ time units in inventory is given by $p e^{-r x}$. For the same unit, the discounted inventory holding cost is given by $h \int_{0}^{x} e^{-r t} d t$, which equals $h\left(1-e^{-r x}\right) / r$. For an item that perishes at some time $x$, the discounted perishing cost becomes $\pi e^{-r x}$. The assumption of constant discount rate can easily be relaxed with time-dependent rates; however, for problems of realistic horizon lengths, discount rates are typically time-invariant. The demand is assumed to be price sensitive and the arrival times of unit demands constitute a renewal process for a fixed selling price. We consider a general model for the price-arrival time relationship and discuss two special cases commonly encountered in literature. The pricing objective is to maximize the expected discounted profit by determining the selling prices dynamically over the horizon for a given initial stocking level $Q$. Without loss of generality, we assume that the beginning of the horizon coincides with a (fictitious) demand arrival.

When the demand process is non-Markovian, the selection of pricing decision epochs becomes important from a modeling perspective. With non-Markovian demands, standard periods of constant length introduce memory and generate correlated demand distributions within periods. Furthermore, if price changes are allowed to be made between two consecutive demand arrivals, it is not clear, in general, how one can adjust the demand arrival time probability distribution for the remaining times. To overcome such difficulties, we choose the instances of demand arrivals as the pricing decision epochs. Hence, inter-demand times are independent but may not be identical random variables.

We assume that there may be a non-zero, fixed cost associated with a price change. Let $p$ and $y$ be the new and the previously set prices, respectively. Then, the price change cost is $C$ if $y \neq p$, and zero, otherwise. Such costs are well-known in the economics literature as menu costs, and correspond to costs incurred to inform the market of the new prices, such as, expenses of advertising and announcement, printing of price tags, catalogs, menus, etc. Herein, we refer to these costs, interchangeably, as menu or advertisement costs. We do not specify a priori any directional restrictions on pricing decisions. Thus, price reversals over the horizon are possible in our initial formulations. The so-called bi-directional pricing 
decisions may indeed be optimal since they allow for the greatest flexibility. However, in practice, decision-makers sometimes restrict price changes to a particular direction-markups or markdowns-only. Although suboptimal from a purely analytical perspective, such uni-directional pricing policies may be based on customer relations or related managerial concerns.

The pricing problem at hand is formulated using a DP approach. A "period" in our formulation corresponds to the time between two consecutive demand occurrences or the expiry of all items on hand, whichever occurs first. As such, it is of random length governed by the demand arrival process and the remaining shelf life of the items. The number of items on hand, say $n$, and the remaining shelf life, say $\tau_{n}$, immediately after a demand arrival constitute the stage. The transition from stage $\left(\tau_{n}, n\right)$ to $\left(\tau_{n}-x, n-1\right)$ occurs with a demand arrival after $x$ time units have elapsed since the beginning of the current period and the transition to stage $(0, n)$ implies that $n$ items on hand have perished at their expiry date (with no demand arriving for $\tau_{n}$ time units). Clearly, the selling prices will be determined on the basis of the units on hand and the remaining shelf life at the beginning of a period.

Let $\Gamma_{n}\left(\tau_{n}, p\right)$ denote the within-period expected discounted profit obtained starting with $n$ items and remaining shelf life of $\tau_{n}$ when the selling price is set at $p$ for the period. Furthermore, let $\Theta_{n}\left(\tau_{n}, y, p\right)$ denote the expected discounted profit when there are $n$ items on hand, the remaining shelf life is $\tau_{n}, y$ is the price immediately after the last demand arrival, and the selling price is set at $p$ for the current period and optimal pricing policy is employed for the remainder of the horizon; the corresponding optimal expected discounted profit is denoted by $\kappa_{n}^{*}\left(\tau_{n}, y\right)$ for convenience. We will denote the cumulative distribution function (c.d.f.) of the general inter-demand time by $G_{u}(x)$ and the complementary c.d.f. by $\bar{G}_{u}(x)$, when price $p=u$ is used. Throughout $\mathbf{1}_{(y \neq p)}$ is the indicator function being equal to unity if $y \neq p$ and zero, otherwise. Then, we have:

$$
\begin{aligned}
\Gamma_{n}\left(\tau_{n}, p\right)= & \int_{0}^{\tau_{n}} p e^{-r x} d G_{p}(x)-\int_{0}^{\tau_{n}} n h \frac{1-e^{-r x}}{r} d G_{p}(x) \\
& -n\left(h \frac{1-e^{-r \tau_{n}}}{r}+\pi e^{-r \tau_{n}}\right) \bar{G}_{p}\left(\tau_{n}\right),
\end{aligned}
$$

where $\tau_{n} \leqslant \tau$ for all $n=1, \ldots, Q$, and

$$
\begin{aligned}
\kappa_{n}^{*}\left(\tau_{n}, y\right)= & \max _{p}\left\{\Theta_{n}\left(\tau_{n}, y, p\right)\right\} \\
= & \max _{y}\left\{-C 1_{(p \neq y)}+\Gamma_{n}\left(\tau_{n}, p\right)\right. \\
& \left.+\int_{0}^{\tau_{n}} \kappa_{n-1}^{*}\left(\tau_{n}-x, p\right) e^{-r x} d G_{p}(x)\right\} .
\end{aligned}
$$

Clearly, $\kappa_{n}^{*}(0, y):=-n \pi$ for all $y$ and $\kappa_{0}^{*}(x, y):=0$ for all $x \leqslant \tau$ and $y$; and we set $\tau_{Q}=\tau$ initially.

Without loss of generality, we assume that, as the problem horizon begins, the "past" price is set at zero. Therefore, the optimal profit for an initial inventory of $Q$ items for a problem horizon of length $\tau$ is given by $\kappa_{Q}^{*}(\tau, 0)$. Correspondingly, the optimal starting price would be $p^{*}$ for given initial stock $Q$ and shelf life $\tau$. (Note that we suppress the indices of state on price for brevity.)

The formulation above allows for various forms of price-dependent demand inter-arrival times. Poisson arrivals with price-dependent rate $\lambda(p)$, which has constant price elasticity, is a common and general example, where $\bar{G}_{p}(x)=e^{-\lambda(p) x}$. Another example would be the Weibull distributed inter-demand times; with $\bar{G}_{p}(x)=$ $e^{-(\lambda(p) x)^{\beta}}$. In both of these examples, the scale parameter $\lambda(p)$ can be any non-negative function decreasing in price. A frequently used example is $\lambda(p)=b-a p$, where $a>0$ and $b$ are constants. In settings where the retailer also makes the stocking decision, we have the joint optimization problem $J^{*}(\tau)=\max _{Q} \kappa_{Q}^{*}(\tau, 0)-v(Q)$, where $v(Q)$ denotes the net present value of the cost of acquiring $Q$ items.

\section{Structural results}

We begin with the result on the concavity of the within-period expected profit with respect to the selling price. (Most of the proofs are based on standard optimization techniques, and are either omitted or briefly sketched herein.)

Proposition 1 (Price-concavity). $\Gamma_{n}\left(\tau_{n}, p\right)$ is concave in $p$ if $\bar{G}_{p}(x)$ is monotonically non-decreasing and strictly convex in $p$.

The conditions on $\bar{G}_{p}(x)$ are satisfied for a number of inter-demand distributions under some mild conditions. For a Poisson demand process with rate $\lambda(p)=b-a p(>0)$, where $G_{p}(x)=e^{-\lambda(p) x}$, the above result holds for all positive $a$ and $b$. For Weibull distributed inter-arrival times with parameters $\lambda(p)$ and $\beta(\geqslant 1)$, where $G_{p}(x)=e^{-\left(\lambda(p)^{x}\right)^{\beta}}$, the above result holds if $\lambda(p)$ is decreasing and strictly concave in $p$ and $\beta$ is an even integer. This property of $\Gamma_{n}\left(\tau_{n}, p\right)$ enables us to specify the structure of the optimal pricing policy for $n=1$. It directly follows that the optimal pricing policy for a single item consists of a three-parameter $\left(p^{*}, p_{L}, p_{U}\right)$ policy which we state below.

Corollary 1 (Policy structures $I$ ). Suppose $\bar{G}_{p}(x)$ is monotonically non-decreasing and strictly convex in $p$. For $n>0$ and any $\tau_{n}$, let $p^{*}\left(\tau_{n}, n\right)$ be the maximizer of $\Gamma_{n}\left(\tau_{n}, p\right) ; \Gamma_{n}^{*}\left(\tau_{n}\right)$ denote the corresponding maximum; $p_{L}\left(\tau_{n}, n\right)$ and $p_{U}\left(\tau_{n}, n\right)$ be the prices such that $\Gamma_{n}\left(\tau_{n}, p_{L}\left(\tau_{n}, n\right)\right)=\Gamma_{n}\left(\tau_{n}, p_{U}\left(\tau_{n}, n\right)\right)=$ $\Gamma_{n}\left(\tau_{n}, p^{*}\left(\tau_{n}, n\right)\right)-C$. For $n=1$ and any $\tau_{1}$, it is optimal to raise the selling price to $p^{*}\left(\tau_{1}, 1\right)$ if the previous price was set either (at or) below $p_{L}\left(\tau_{1}, 1\right)$ or (at or) above $p_{U}\left(\tau_{1}, 1\right)$; otherwise, it is optimal to keep the price in place.

The optimal policy above consists of three regions: where the price is raised to a maximizer, where no price change is optimal and where the price is reduced to a maximizer. In Fig. 1, we plot the region of no price change (between $p_{L}\left(\tau_{1}, 1\right)$ and $\left.p_{U}\left(\tau_{1}, 1\right)\right)$ for $n=1$ as an example. Note that the lower and upper price change limits, $p_{L}\left(\tau_{1}, 1\right)$ and $p_{U}\left(\tau_{1}, 1\right)$, appear to be non-decreasing in $\tau_{1}$; also note that the difference between the two limits appears to be decreasing in $\tau_{1}$. We have not been able to 


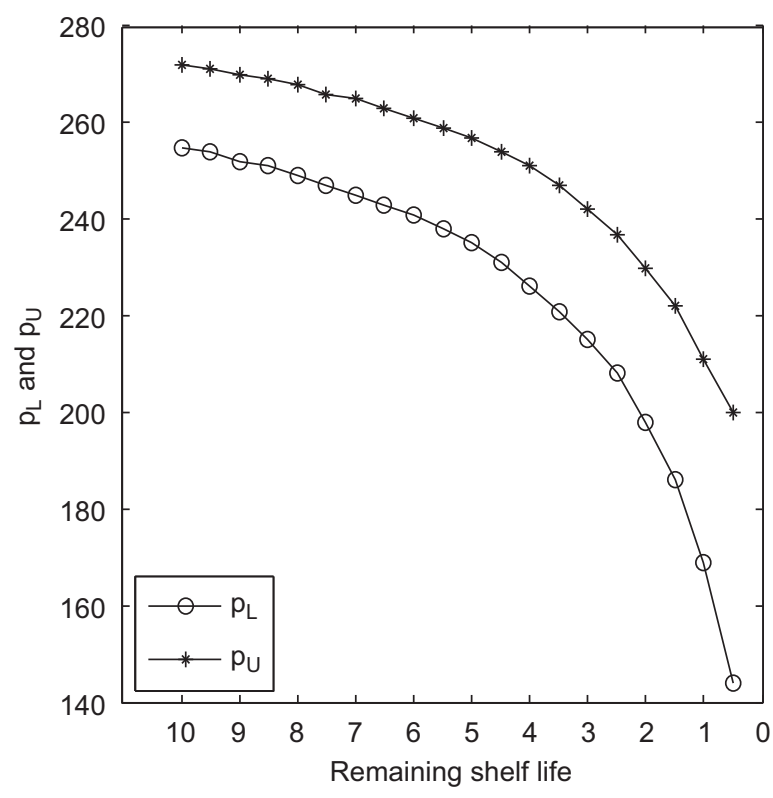

Fig. 1. Limits of no price change region between $p_{L}\left(\tau_{1}, 1\right)$ and $p_{U}\left(\tau_{1}, 1\right)$ for $n=1$ (Poisson demands with $\lambda(p)=b-a p, a=0.01, b=3, \pi=5$, $r=0.01$, and $C=3)$.

demonstrate that the above three-parameter policy is optimal for $n>1$. However, it is the optimal myopic policy.

Corollary 2 (Policy structures II). Suppose $\bar{G}_{p}(x)$ is monotonically non-decreasing and strictly convex in $p$. For $n>0$ and any $\tau_{n}$, let $p^{*}\left(\tau_{n}, n\right)$ be the maximizer of $\Gamma_{n}\left(\tau_{n}, p\right) ; \Gamma_{n}^{*}\left(\tau_{n}\right)$ denote the corresponding maximum; $p_{L}\left(\tau_{n}, n\right)$ and $p_{U}\left(\tau_{n}, n\right)$ be the prices such that $\Gamma_{n}\left(\tau_{n}, p_{L}\left(\tau_{n}, n\right)\right)=\Gamma_{n}\left(\tau_{n}, p_{U}\left(\tau_{n}, n\right)\right)=$ $\Gamma_{n}\left(\tau_{n}, p^{*}\left(\tau_{n}, n\right)\right)-C$. For $n>1$ and any $\tau_{n}$, it is myopically optimal to raise the selling price to $p^{*}\left(\tau_{n}, n\right)$ if the previous price was set either (at or) below $p_{L}\left(\tau_{n}, n\right)$ or (at or) above $p_{U}\left(\tau_{n}, n\right)$; otherwise, it is optimal to keep the price in place.

In our numerical results, we observed that there is a single $\left(p_{L}\left(\tau_{n}, n\right), p_{U}\left(\tau_{n}, n\right)\right)$ pair for all $\tau_{n}$ and $n$. The behavior for these two limits observed for $n=1$ also holds in our experiments for all $n$.

Next we consider some properties of the problem with respect to the remaining shelf life, $\tau_{n}$. To this end, we state below a boundedness condition for the inter-demand arrival distribution.

Condition 1 (Hazard rate boundedness). The hazard rate of the inter-demand arrival distribution $G_{p}(x)$ satisfies the following boundedness condition: $g_{p}\left(\tau_{n}\right) / \bar{G}_{p}\left(\tau_{n}\right)>n(h-\pi r) /$ $(p+n \pi)$.

This condition is always satisfied if $\pi>h / r$, which has an intuitive interpretation: if the cost of perishing for a unit is more expensive than holding the unit in stock forever, then, the expected discounted profit is increasing in the shelf life for all demand distributions. Otherwise, the result may not always hold. For Poisson demand processes, it holds if prices are selected within a certain range to guarantee the arrival rate to be greater than a particular value. For Weibull inter-demand times, the condition gets more stringent and less likely to hold: $\beta[\lambda(p)]^{\beta} \tau_{n}^{(\beta-1)}$ $>n(h-\pi r) /(p+n \pi)$.

Condition 2 (Shape condition). The shape of the interdemand arrival probability density function $g_{p}(x)$ satisfies the following boundedness condition: $\left.\frac{d}{d x} g_{p}(x)\right|_{x=\tau_{n}} /-g_{p}\left(\tau_{n}\right)>n(h-\pi r) /(p+n \pi)$.

If the inter-demandarrival distribution has its hazard rate and shape bounded as defined in the above conditions, the within-period expected profit has some monotonicity and concavity properties with respect to the remaining shelf life. We state these below.

Proposition 2 (Shelf life monotonicity and concavity within period). (a) Suppose only Condition 1 holds. Then, $\Gamma_{n}\left(\tau_{n}, p\right)$ is strictly increasing in $\tau_{n}$ for a given $p$ for all $\tau_{n} \leqslant \tau$ and $n=1,2, \ldots, Q$. (b) Suppose both Conditions 1 and 2 hold. Then, $\Gamma_{n}\left(\tau_{n}, p\right)$ is concave in $\tau_{n}$ for a given $p$ for all $\tau_{n} \leqslant \tau$ and $n=1,2, \ldots, Q$.

Note that the conditions above are sufficiency conditions for the within-period profit to be concave in the shelf life. This result is consistent with the numerical observations we had above on the behavior of the limits of the "no price change"-region for $n=1$ over $\tau_{1}$, although the above conditions were not necessarily satisfied in that particular instance. It has been shown previously that the expected discounted profit (or revenue) is non-decreasing and concave in horizon length under continuous pricing (Gallego and van Ryzin, 1994; Zhao and Zheng, 2000). Below, we show that this property holds in our setting under some (restrictive) conditions.

Proposition 3 (Horizon length sensitivity). Suppose both Conditions 1 and 2 hold. Then, for $\pi=0, \kappa_{n}^{*}\left(\tau_{n}, p\right)$ is monotonically non-decreasing in $\tau_{n}$ for all $p, \tau_{n} \leqslant \tau$ and $n=1,2, \ldots, Q$.

Proof. The construction of the proof is based on the definition of a derivative and is sketched briefly below. Let $p^{*}(x, n, y)$ denote the maximizer of $\Theta_{n}(x, y, p)$.

$$
\begin{aligned}
\frac{\partial}{\partial \tau_{n}} \kappa_{n}^{*}\left(\tau_{n}, y\right) & =\lim _{\delta \rightarrow 0} \frac{\kappa_{n}^{*}\left(\tau_{n}+\delta, y\right)-\kappa_{n}^{*}\left(\tau_{n}, y\right)}{\delta} \\
& =\lim _{\delta \rightarrow 0} \frac{\Theta_{n}\left(\tau_{n}+\delta, y, p^{*}\left(\tau_{n}+\delta, n, y\right)\right)-\Theta_{n}\left(\tau_{n}, y, p^{*}\left(\tau_{n}, n, y\right)\right)}{\delta} \\
& \geqslant \lim _{\delta \rightarrow 0} \frac{\Theta_{n}\left(\tau_{n}+\delta, y, p^{*}\left(\tau_{n}, n, y\right)\right)-\Theta_{n}\left(\tau_{n}, y, p^{*}(\tau, n, y)\right)}{\delta} \\
& =\left.\frac{\partial}{\partial \tau_{n}} \Theta_{n}\left(\tau_{n}, y, p\right)\right|_{p=p^{*}\left(\tau_{n}, n, y\right)} \geqslant 0 .
\end{aligned}
$$

The first inequality follows from the fact that $y^{*}(t, n, p)$ may not be optimal. Taking the limit, we get the first inequality that $\partial / \partial \tau_{n} \kappa_{n}^{*}\left(\tau_{n}, p\right) \geqslant \partial /\left.\partial \tau_{n} \kappa_{n}\left(\tau_{n}, y\right)\right|_{y=y^{*}\left(\tau_{n}, n, p\right)}$. To establish the last inequality, we proceed inductively. From Proposition 2, we have $\Gamma_{n}\left(\tau_{n}, p\right)$ is concave in $\tau_{n}$ for all $n$ and $p$. Clearly, this holds for $n=1$; and implies that, if $\kappa_{1}^{*}\left(\tau_{1}, p\right)$ is non-decreasing in $\tau_{1}$, then so is $\kappa_{2}^{*}\left(\tau_{2}, p\right)$. Suppose $\kappa_{n}^{*}\left(\tau_{n}, p\right)$ is non-decreasing in $\tau_{n}$ for $n>1$, then the last inequality holds. The result follows inductively.

Hence, in the pricing problem where price decision epochs are restricted to coincide with demand arrivals, 
one cannot always guarantee monotone behavior and/or concavity with respect to the shelf lives of items. Although the monotonicity result above is subject to certain conditions, we have observed that the expected discounted profit is monotone and non-decreasing for the cases we examined via numerical analysis (e.g., Fig. 6 (b)).

\section{Numerical study}

In the experiments reported herein, we assume that demands are generated according to a Poisson process with a price-sensitive rate $\lambda(p)=b-a p$. We considered $a=0.01,0.05,0.10, b=3,4, \pi=5,10,20, C=0,5,10,25$, $r=0.01,0.1,0.25,0.5$. We set $h=1$ and varied $\tau$ between 0 and 10 . We have taken acquisition costs to be linear, $v(Q)=c_{o} Q$ where $c_{o}=0,0.3 / a$. The optimal pricing policy obtained from the DP formulation provides a list of prices as demands occur over time for every state of the system given by $\left(n, \tau_{n}, y\right)$ as defined before. In the absence of a known optimal policy class, in order to obtain the optimal dynamic pricing solutions, we used exhaustive search over the permissible price range with increments of 0.01 and 1 . Preliminary studies indicate that the goodness of the solution does not depend much on the size of the search increment; but, the solution time is significantly dependent on it. (The same search routine was used for the heuristics, which are discussed later, except for Heuristics $I$ and $I V$, for which the golden section search method was employed.) Problem horizon length was discretized by increments of 0.01 time units.

\subsection{Sensitivity analysis}

It is interesting to see how the prices and the corresponding values of the expected profit-to-go function evolve over the problem horizon. However, it is impossible to list all the pricing decisions for all possible demand realization instances. Hence, in order to examine the resulting price and profit-to-go profiles, we instead highlight three demand realization sample paths among all possible sequences considered in the solution: arrivals occurring early in the horizon, arrivals grouped in the middle of the horizon and, lastly, arrivals coming later in the horizon. The pricing profiles of interest are the optimal prices chosen for that state (i.e., the number on hand and the remaining shelf life) after each demand occurs and a a

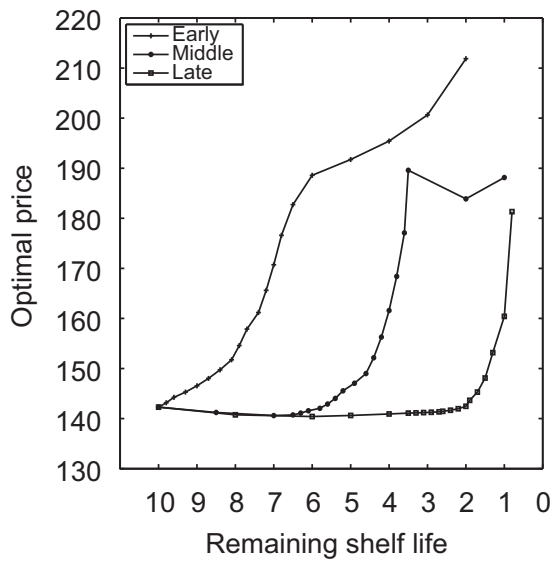

b

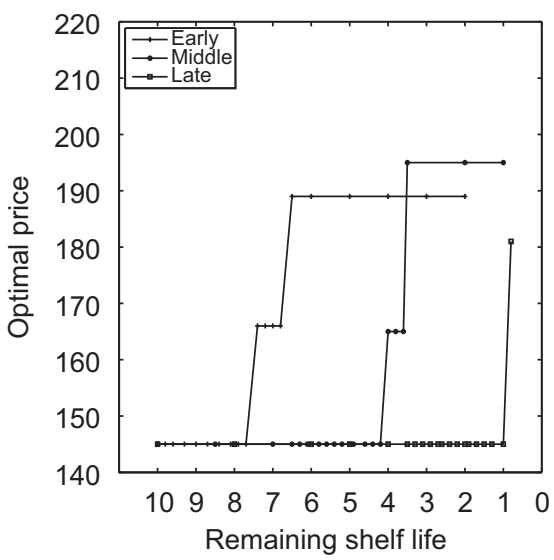

C

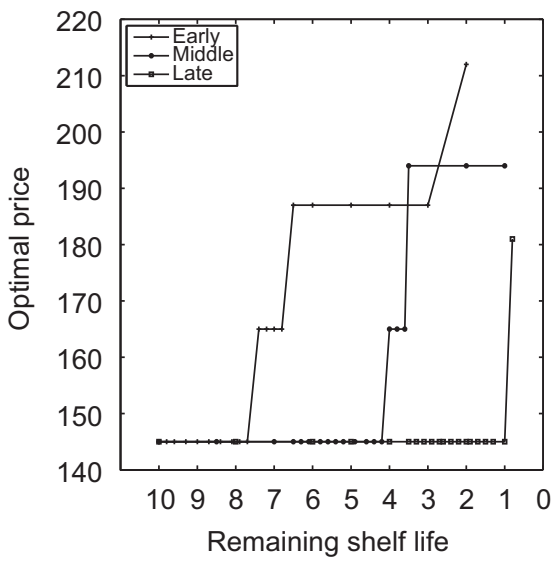

Fig. 2. Optimal pricing trajectories for early-middle-late arrival patterns $(a=0.01, b=3, \pi=15, r=0.1$ ): (a) no menu cost, $C=0$; (b) positive menu cost, $C=5$ and bidirectional price change allowed; (c) positive menu cost, $C=5$ and markups only. 
unit is sold. Each pricing and profit-to-go profile gives an indication of the responsiveness of the problem to changes in its state. The price profile directly illustrates the price ranges considered to manage the demand, as well. We present some examples below for different system and cost parameters to gain insights about the pricing dynamics of the problem.
In Fig. 2 (a), we illustrate a case with no price change cost. This case constitutes a benchmark for two reasons. First, it enables the maximum profit due to complete freedom of price changes, and hence, it provides an upper bound on the objective function. Second, it is the cost structure that has been previously considered in the literature. With zero menu costs and continuous review,

a
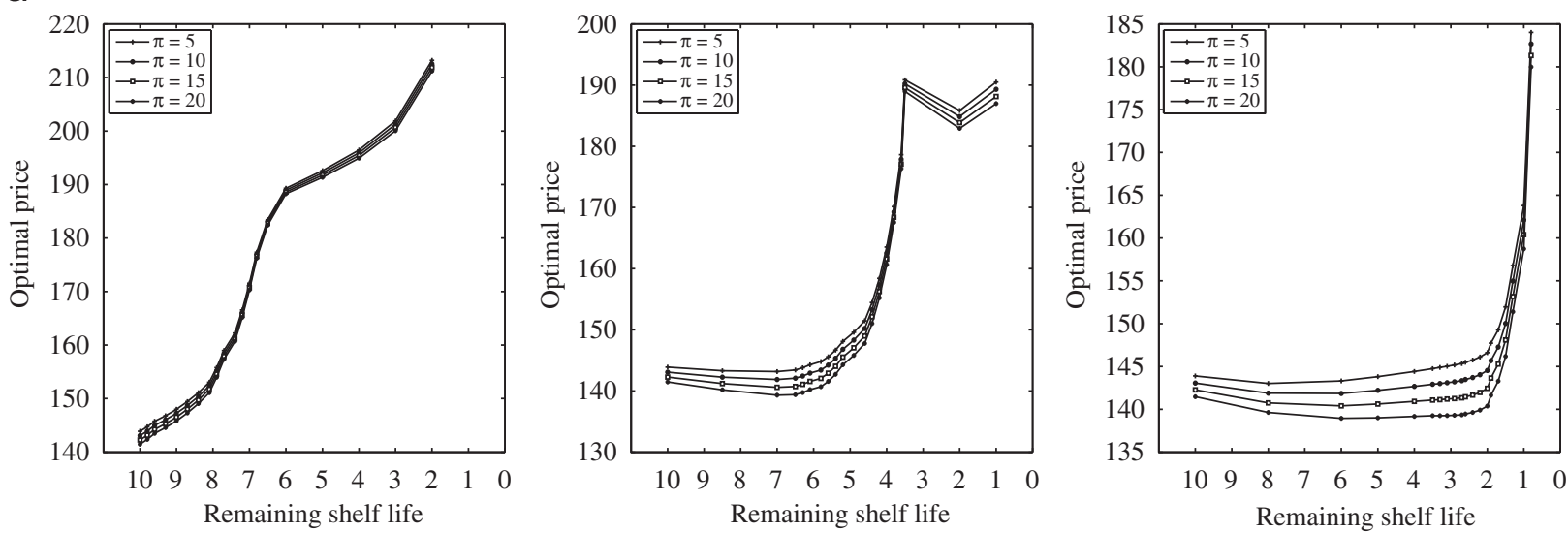

b


C
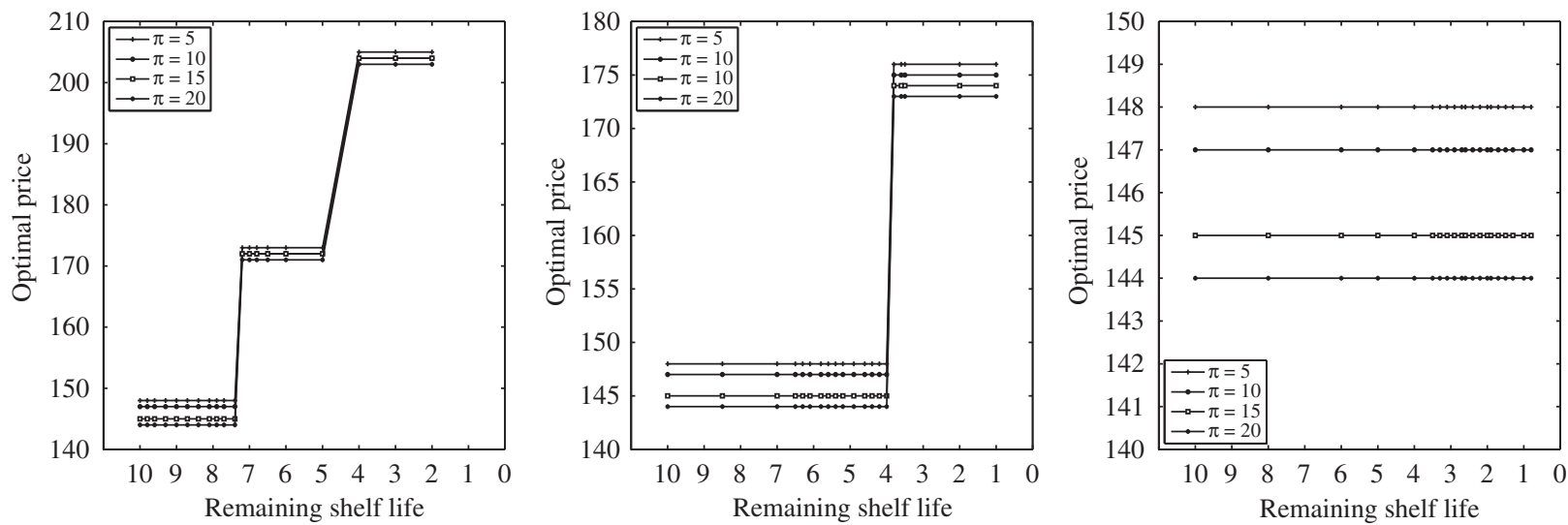

Fig. 3. Impact of $\pi$ on price profiles for early-middle-late arrival patterns $(a=0.01, b=3, r=0.1$ ): (a) zero menu costs, $C=0$; (b) positive menu costs, $C=5$, and bidirectional price changes allowed; (c) positive menu costs, $C=10$, and bidirectional price changes allowed. 
from Gallego and van Ryzin (1994), we know that prices exhibit the following monotone behavior: for any given remaining lifetime, prices are decreasing in the number on hand. Our numerical experiment differs from their setting in that we restrict price change decisions to coincide with demand arrival epochs. The overall behavior of pricing decisions in our results supports their finding. As arrivals occur, the pricing tends to get more aggressive.
Yet, the remaining time in the horizon also plays an important role. This is most apparent in the middle realization. Here, there is aggressive pricing as demands occur in rapid succession but prices are reduced drastically as one approaches the end of the horizon to lessen the costs of perishing.

In Fig. 2 (b), we present the corresponding price profiles when a positive price change cost (menu or

a
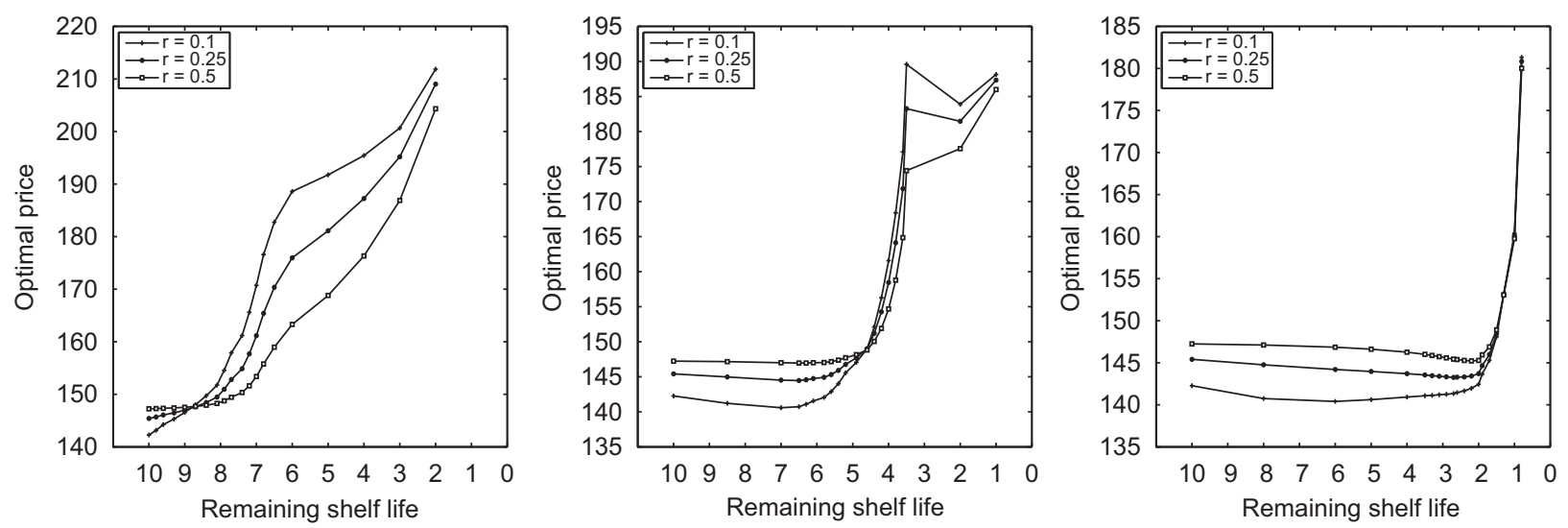

b
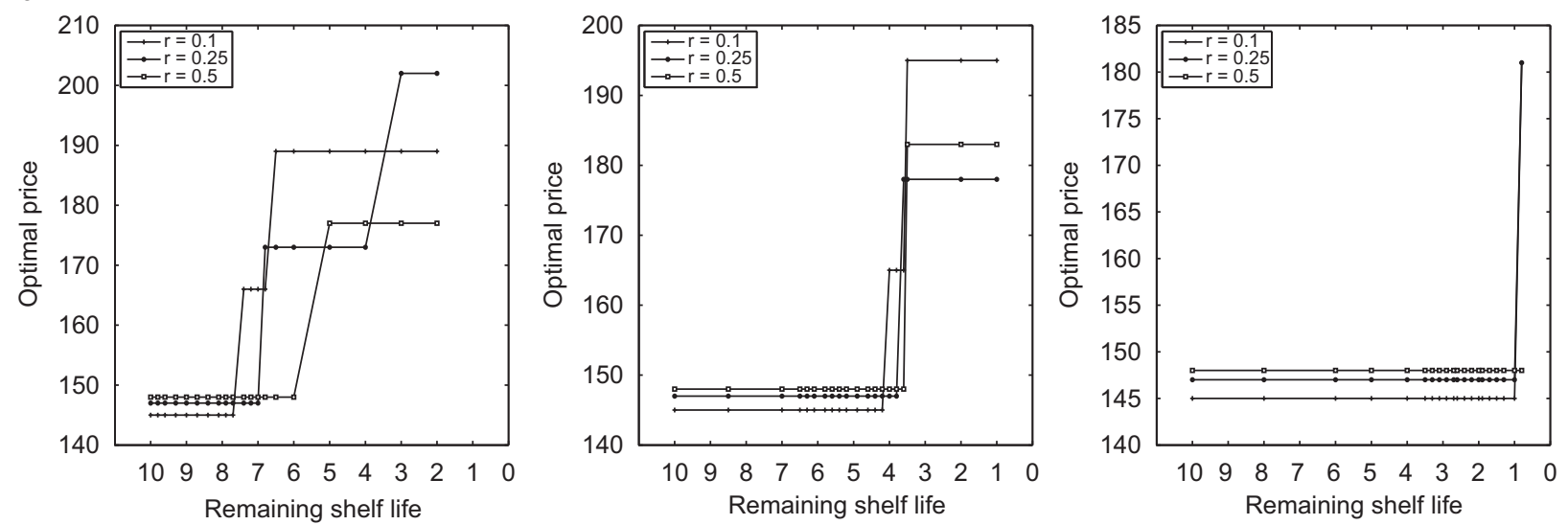

C
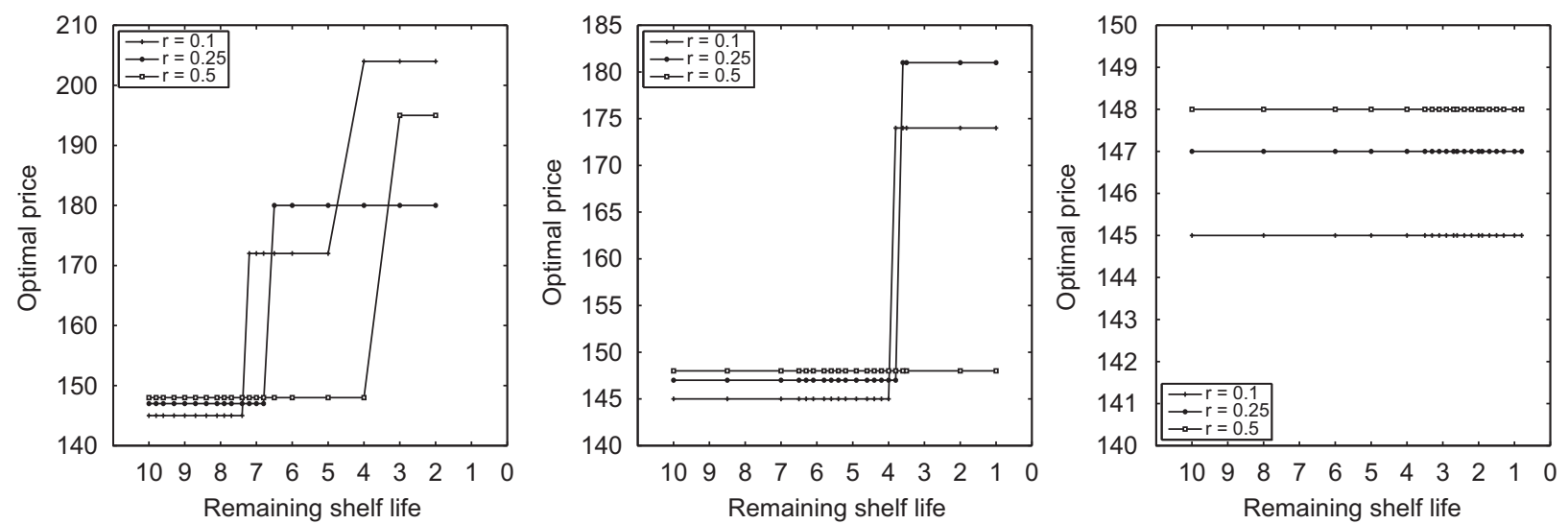

Fig. 4. Impact of $r$ on price profiles for early-middle-late arrival patterns $(a=0.01, b=3, \pi=15)$ : (a) zero menu costs, $C=0$; (b) positive menu costs, $C=5$, and bidirectional price changes allowed; (c) positive menu costs, $C=10$, and bidirectional price changes allowed. 
advertising cost) is introduced. When changes are costly, there is much less nervousness in pricing, as expected, even for a modest value of menu costs. As the price change cost increases, the number of price change decisions quickly diminishes over the horizon. Since price changes are costly, they are reserved for actions that may bring in the most contribution to compensate for the fixed menu costs. As fewer price changes become desirable, so do upward price movements. In the profiles shown, all price movements are in one direction (upwards) over the horizon although we allow for bi-directional movements. The timing of these price changes also exhibits a lag compared to the case of no menu costs;price change decisions are postponed to reduce their cost impact through discounting.

In Fig. 2 (c), we give the corresponding profiles when we deliberately restrict the price movement to one direction-markups only. Comparing with the bidirectional case, we do not see discernible difference for the sample paths when arrivals group in the middle and later in the horizon. But when demands come early in the horizon, pricing is more aggressive. This again can be
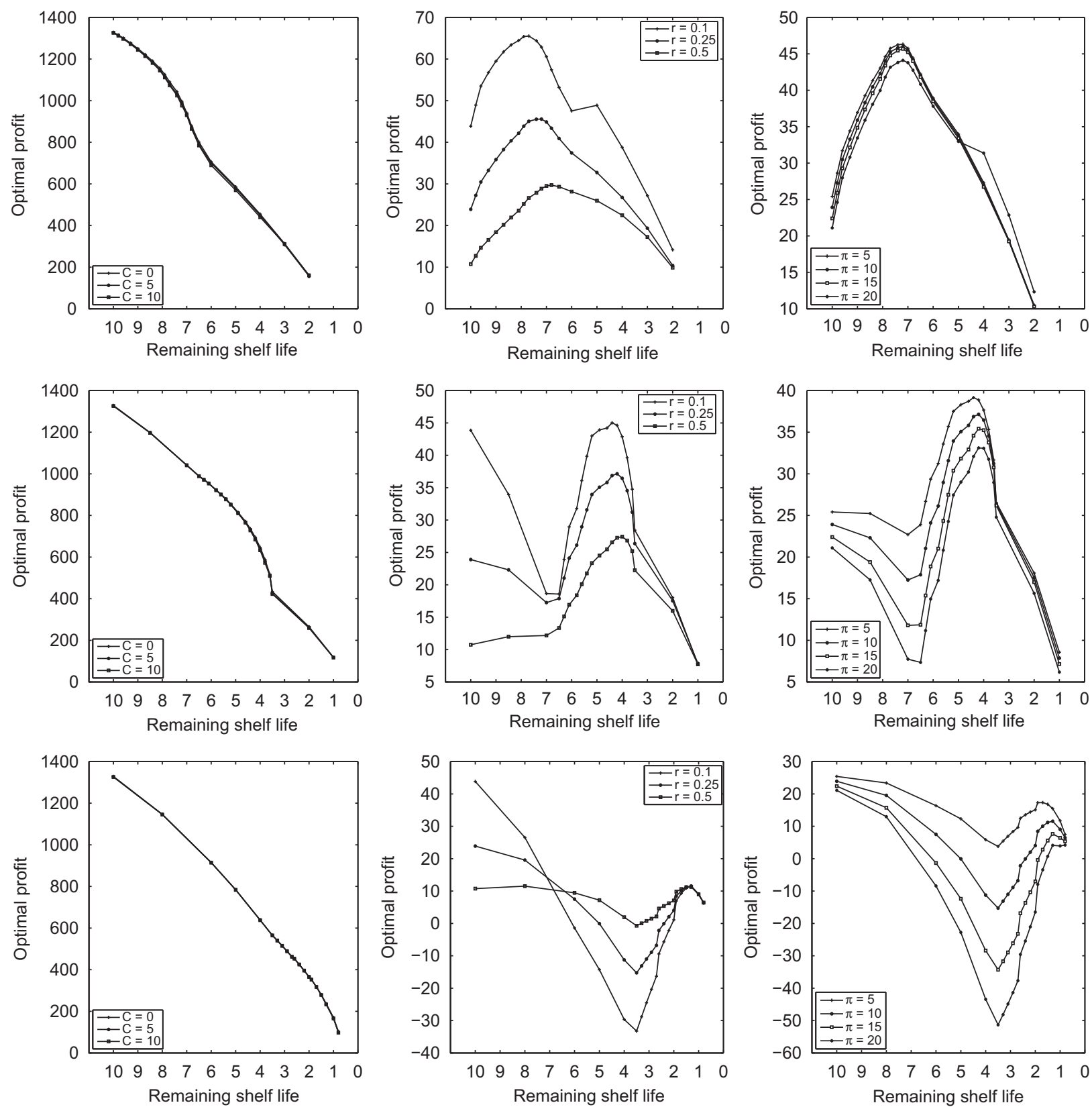

Fig. 5. Impact of system parameters on expected profit-to-go function values for early-middle-late arrival patterns. (Left: $a=0.01, b=3, \pi=10, r=0.10$; center: $a=0.10, b=3, \pi=10, C=10$; right: $a=0.10, b=3, r=0.25, C=5$ ). 
explained through the lessened impact of perishing toward the end of the horizon.

When we considered only markdowns, we saw that, in all three arrival patterns, a single price (150) was selected throughout! This behavior is consistent with the above observations. As demands occur (and, by definition, the remaining shelf life gets smaller), higher prices are desired; if they are not permissible, then a trade off is achieved between losing some early sales but gaining later in the horizon with higher prices.

In Figs. 3-5, we (i) give further examples to highlight the impacts of the unit perishing cost $\pi$, and the discount rate $r$, with and without price change costs on the dynamic price profiles, and (ii) plot the profit-to-go function for certain scenarios to illustrate its sensitivity with respect to various system parameters. Fig. 3 shows that the impact of $\pi$ is not significant on the optimal price profile when price changes are not costly. When price changes are costly, differentiation emerges. The introduc- tion of positive price change costs results in a decrease in the number of price changes over the horizon, as expected. For the arrival pattern with late arrivals, we see the price profile is that of a single price. However, the impact of higher unit perishing costs is not discernible on the profit-to-go function values when price change cost is zero. The insensitivity of the profit-to-go is consistent with one's expectation that optimal pricing would attempt to reduce perishing to minimize costs.

As the menu cost is introduced, however, there may be some cases where the sensitivity of the expected profitto-go function is significant (e.g., Fig. 5). The impact of the discount rate is quite large on the actual pricing decisions, as expected (see Fig. 4). It is interesting to note that the direction of the pricing decisions is similar in the portions of problem horizon where demand groupings occur regardless of the discount rate. The introduction of positive price change costs results in a decrease in the number of price changes over the horizon in this case as

Table 1

Sensitivity results w.r.t. system parameters for 1 and 5 items.

\begin{tabular}{|c|c|c|c|c|c|c|c|c|c|c|c|c|c|c|}
\hline \multirow[t]{3}{*}{$b$} & \multirow[t]{3}{*}{$a$} & \multirow[t]{3}{*}{$\tau$} & \multicolumn{6}{|l|}{1 Item } & \multicolumn{6}{|l|}{5 Items } \\
\hline & & & \multicolumn{2}{|l|}{$\pi=5$} & \multicolumn{2}{|l|}{$\pi=10$} & \multicolumn{2}{|l|}{$\pi=20$} & \multicolumn{2}{|l|}{$\pi=5$} & \multicolumn{2}{|l|}{$\pi=10$} & \multicolumn{2}{|l|}{$\pi=20$} \\
\hline & & & $p^{*}$ & $\kappa^{*}$ & $p^{*}$ & $\kappa^{*}$ & $p^{*}$ & $\kappa^{*}$ & $p^{*}$ & $\kappa^{*}$ & $p^{*}$ & $\kappa^{*}$ & $p^{*}$ & $\kappa^{*}$ \\
\hline \multirow{15}{*}{3} & \multirow{5}{*}{0.01} & 0.5 & 172.52 & 76.65 & 170.84 & 74.14 & 167.51 & 69.20 & 146.73 & 87.77 & 144.32 & 67.65 & 139.51 & 27.58 \\
\hline & & 2.5 & 220.64 & 173.43 & 220.10 & 172.90 & 219.04 & 171.85 & 155.91 & 453.80 & 154.38 & 447.01 & 151.38 & 433.75 \\
\hline & & 5.0 & 240.58 & 197.97 & 240.36 & 197.82 & 239.94 & 197.51 & 181.78 & 667.80 & 181.26 & 665.91 & 180.24 & 662.25 \\
\hline & & 7.5 & 248.37 & 204.37 & 248.28 & 204.32 & 248.09 & 204.22 & 197.18 & 743.18 & 196.99 & 742.56 & 196.63 & 741.37 \\
\hline & & 10.0 & 251.60 & 206.19 & 251.56 & 206.17 & 251.49 & 206.15 & 204.11 & 769.19 & 204.05 & 768.98 & 203.92 & 768.58 \\
\hline & \multirow{5}{*}{0.05} & 0.5 & 33.09 & 13.07 & 31.46 & 10.69 & 28.35 & 6.21 & 0 & - & 0 & - & 0 & - \\
\hline & & 2.5 & 43.44 & 33.49 & 42.95 & 33.01 & 42.04 & 32.14 & 29.40 & 79.59 & 27.99 & 73.53 & 25.44 & 62.71 \\
\hline & & 5.0 & 47.56 & 38.38 & 47.37 & 38.25 & 47.01 & 38.00 & 35.06 & 123.76 & 34.62 & 122.19 & 33.85 & 119.40 \\
\hline & & 7.5 & 49.10 & 39.58 & 49.02 & 39.54 & 48.87 & 39.47 & 38.17 & 138.31 & 38.02 & 137.83 & 37.77 & 136.96 \\
\hline & & 10.0 & 49.70 & 39.90 & 49.67 & 39.89 & 49.61 & 39.87 & 39.46 & 142.98 & 39.41 & 142.83 & 39.33 & 142.55 \\
\hline & \multirow{5}{*}{0.10} & 0.5 & 15.68 & 5.17 & 14.12 & 2.93 & 0 & - & 0 & - & 0 & - & 0 & - \\
\hline & & 2.5 & 21.32 & 16.03 & 20.87 & 15.61 & 20.10 & 14.89 & 13.64 & 33.21 & 12.38 & 27.92 & 10.30 & 19.34 \\
\hline & & 5.0 & 23.45 & 18.47 & 23.28 & 18.36 & 22.99 & 18.16 & 16.77 & 56.06 & 16.41 & 54.78 & 15.85 & 52.71 \\
\hline & & 7.5 & 24.21 & 19.02 & 24.14 & 18.99 & 24.02 & 18.94 & 18.33 & 63.00 & 18.22 & 62.63 & 18.04 & 62.02 \\
\hline & & 10.0 & 24.48 & 19.16 & 24.46 & 19.15 & 24.42 & 19.13 & 18.91 & 65.04 & 18.88 & 64.94 & 18.82 & 64.75 \\
\hline \multirow{15}{*}{4} & \multirow{5}{*}{0.01} & 0.5 & 239.61 & 126.86 & 238.10 & 124.74 & 235.12 & 120.54 & 195.64 & 175.39 & 193.25 & 156.46 & 188.46 & 118.78 \\
\hline & & 2.5 & 307.98 & 254.82 & 307.57 & 254.43 & 306.75 & 253.67 & 220.78 & 769.95 & 219.62 & 765.20 & 217.34 & 755.92 \\
\hline & & 5.0 & 332.20 & 282.22 & 332.05 & 282.12 & 331.75 & 281.92 & 262.18 & 1038.36 & 261.86 & 1037.23 & 261.24 & 1035.01 \\
\hline & & 7.5 & 340.77 & 288.46 & 340.71 & 288.43 & 340.59 & 288.38 & 280.24 & 1115.60 & 280.14 & 1115.28 & 279.96 & 1114.64 \\
\hline & & 10.0 & 343.87 & 289.96 & 343.85 & 289.96 & 343.81 & 289.94 & 286.73 & 1137.85 & 286.70 & 1137.76 & 286.65 & 1137.57 \\
\hline & \multirow{5}{*}{0.05} & 0.5 & 46.65 & 23.43 & 45.19 & 21.40 & 42.39 & 17.55 & 37.11 & 18.25 & 0 & - & 0 & - \\
\hline & & 2.5 & 61.04 & 49.95 & 60.65 & 49.59 & 59.93 & 48.93 & 42.70 & 144.94 & 41.64 & 140.66 & 39.71 & 132.91 \\
\hline & & 5.0 & 65.97 & 55.37 & 65.83 & 55.28 & 65.56 & 55.11 & 51.37 & 199.17 & 51.10 & 198.19 & 50.61 & 196.42 \\
\hline & & 7.5 & 67.65 & 56.54 & 67.59 & 56.52 & 67.49 & 56.48 & 54.92 & 213.91 & 54.84 & 213.64 & 54.69 & 213.15 \\
\hline & & 10.0 & 68.22 & 56.80 & 68.20 & 56.80 & 68.17 & 56.79 & 56.10 & 217.88 & 56.08 & 217.81 & 56.04 & 217.67 \\
\hline & \multirow{5}{*}{0.10} & 0.5 & 22.55 & 10.54 & 21.15 & 8.62 & 18.54 & 5.15 & 0 & - & 0 & - & 0 & - \\
\hline & & 2.5 & 30.18 & 24.36 & 29.83 & 24.04 & 29.20 & 23.48 & 20.49 & 67.07 & 19.54 & 63.28 & 17.96 & 56.97 \\
\hline & & 5.0 & 32.70 & 27.04 & 32.57 & 26.97 & 32.35 & 26.83 & 25.05 & 94.46 & 24.82 & 93.64 & 24.45 & 92.26 \\
\hline & & 7.5 & 33.51 & 27.58 & 33.47 & 27.56 & 33.39 & 27.53 & 26.77 & 101.40 & 26.71 & 101.19 & 26.60 & 100.82 \\
\hline & & 10.0 & 33.77 & 27.69 & 33.76 & 27.69 & 33.74 & 27.68 & 27.30 & 103.13 & 27.28 & 103.08 & 27.25 & 102.98 \\
\hline
\end{tabular}

Dynamic pricing with no menu costs $(r=0.1)$. 
a

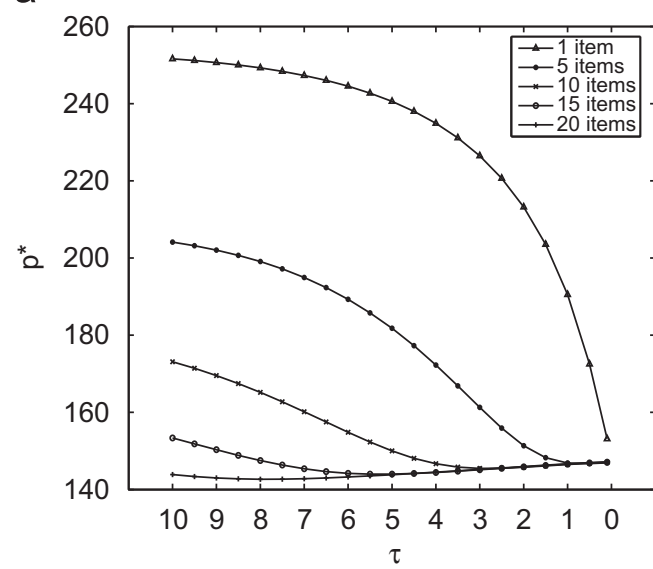

b



Fig. 6. Optimal starting prices and expected discounted profit versus remaining shelf life $(a=0.01, b=3, \pi=5, r=0.10, C=0)$.

well. The expected profit-to-go function is also sensitive to the discount rate (e.g., Fig. 5). Both $\pi$ and $r$ impact the profit-to-go function in a way to exacerbate the inherent trends; that is, a decrease or an increase is exaggerated as either $\pi$ or $r$ increases. Overall, we should point out the highly non-linear behavior of the expected profit-to-go function with respect to remaining shelf life at every demand arrival instance. This is due to the changes in both the remaining shelf lives and the number of units on hand at each decision epoch. The sudden changes are most commonly observed for the late arrival pattern and for high values of $r$ and $C$. Although the overall behavior of $\kappa^{*}(\tau, 0)$ with respect to $\tau$ was numerically observed to be smooth and non-decreasing, the profit-to-go functions at each demand instance are not smooth and not monotone.

Having examined the components, we now turn to the entire optimization problem. In Table 1 , we display some instances to illustrate the overall sensitivity of the optimal starting price and the expected discounted profit to various system parameters. (See also Fig. 6.)

The optimal starting price is increasing in $\tau$, the base demand level $b$, and is decreasing in price sensitivity $a$, unit perishing cost $\pi$ and initial stock size, $Q$. The optimal expected discounted profit is increasing in $\tau$, the base demand level $b$ and initial stock size $Q$, and is decreasing in price sensitivity $a$ and unit perishing cost $\pi$. The results are intuitive and support the theoretical properties discussed above.

When we consider joint optimization of initial stock and pricing, for every horizon length, there is an optimal initial stocking level, $Q^{*}$, that maximizes the expected discounted profit; $Q^{*}=\arg \max _{Q} \kappa_{Q}^{*}(\tau, 0)$. This level is important for a retailer that needs to allocate shelf space for individual products. In Table 2, we present, for different system parameters, the optimal initial stocking levels, the optimal expected discounted profits and the corresponding optimal starting prices.

The optimal initial stock at a given remaining lifetime is non-increasing in the slope of the demand rate $a$ when everything else is fixed. Particularly, when the demand rate decreases, the optimal initial stock decreases if the remaining lifetime is long enough so that a demand is likely to occur. This result is similar for the base demand rate, $b$. Also, $Q^{*}$ is non-decreasing in the remaining lifetime. For any stocking level, the optimal starting price and the expected discounted profit is increasing in the remaining lifetime. The latter is an important numerical observation because it holds only under specific conditions as discussed above. Similar observations hold for positive acquisition and price change costs, as well.

\subsection{Proposed policy heuristics}

From a practitioner's perspective, easily implementable heuristics may be as desirable as the optimal solution, if not more so given that the optimal pricing policy can be found only through exhaustive numerical search. Hence, we propose four implementable policy heuristics and examine their performances with respect to the optimal solution. Each heuristic is based on an approximation of the optimization problem given in Eq. (2) and uses the optimal pricing for the corresponding approximate problem.

Constant pricing policies have been used as a benchmark although they are clearly suboptimal vis a vis an unrestricted policy, (e.g. Rajan et al., 1992; Gallego and van Ryzin, 1994; Federgruen and Heching, 1999; Feng and Xiao, 2000a). In the case of Markovian demands, the fixed price heuristic has been shown to be asymptotically optimal for undiscounted revenue maximization with zero unit holding costs when the number of items to sell is large and the remaining shelf life is accordingly long. A similar result holds in the case of non-Markovian demands as stated below.

Proposition 4 (Asymptotically optimal single price heuristic). For $r=h=0$, a single price (constant pricing) policy heuristic is asymptotically optimal as $\tau_{n} \longrightarrow \infty$ for all $n$. 
Table 2

Optimal starting price, initial stock and expected profit.

\begin{tabular}{|c|c|c|c|c|c|c|c|c|c|c|c|}
\hline \multirow[t]{2}{*}{$b$} & \multirow[t]{2}{*}{$a$} & \multirow[t]{2}{*}{$\tau$} & \multicolumn{3}{|c|}{$\pi=5$} & \multicolumn{3}{|c|}{$\pi=10$} & \multicolumn{3}{|c|}{$\pi=20$} \\
\hline & & & $Q^{*}$ & $p^{*}$ & $\kappa^{*}$ & $Q^{*}$ & $p^{*}$ & $\kappa^{*}$ & $Q^{*}$ & $p^{*}$ & $\kappa^{*}$ \\
\hline \multirow{15}{*}{3} & \multirow{5}{*}{0.01} & 0.5 & 3 & 147.84 & 96.90 & 2 & 150.45 & 89.90 & 2 & 146.09 & 77.50 \\
\hline & & 2.5 & 7 & 147.82 & 470.13 & 7 & 145.93 & 457.28 & 6 & 145.54 & 438.26 \\
\hline & & 5.0 & 11 & 147.48 & 840.17 & 11 & 146.12 & 829.02 & 10 & 146.35 & 809.68 \\
\hline & & 7.5 & 15 & 146.34 & 1123.95 & 15 & 145.33 & 1114.49 & 14 & 145.59 & 1098.20 \\
\hline & & 10.0 & 19 & 145.04 & 1339.50 & 18 & 145.84 & 1331.86 & 18 & 144.50 & 1319.06 \\
\hline & \multirow{5}{*}{0.05} & 0.5 & 2 & 28.68 & 13.63 & 1 & 31.46 & 10.69 & 1 & 28.35 & 6.21 \\
\hline & & 2.5 & 5 & 29.40 & 79.59 & 5 & 27.99 & 73.53 & 4 & 28.09 & 66.16 \\
\hline & & 5.0 & 9 & 28.70 & 141.67 & 8 & 29.06 & 137.26 & 7 & 29.50 & 130.15 \\
\hline & & 7.5 & 11 & 29.61 & 185.35 & 11 & 29.12 & 182.20 & 10 & 29.59 & 177.03 \\
\hline & & 10.0 & 14 & 29.12 & 214.59 & 14 & 28.81 & 212.36 & 13 & 29.23 & 209.31 \\
\hline & \multirow{5}{*}{0.10} & 0.5 & 1 & 15.68 & 5.17 & 1 & 14.12 & 2.93 & 1 & 0 & - \\
\hline & & 2.5 & 4 & 14.77 & 33.97 & 4 & 13.74 & 30.51 & 3 & 14.17 & 26.38 \\
\hline & & 5.0 & 7 & 14.69 & 60.08 & 7 & 14.15 & 57.48 & 6 & 14.54 & 54.25 \\
\hline & & 7.5 & 9 & 14.93 & 76.16 & 9 & 14.67 & 74.77 & 9 & 14.30 & 72.59 \\
\hline & & 10.0 & 11 & 14.76 & 85.24 & 11 & 14.63 & 84.48 & 11 & 14.44 & 83.29 \\
\hline \multirow{15}{*}{4} & \multirow{5}{*}{0.01} & 0.5 & 3 & 198.82 & 181.06 & 3 & 196.51 & 171.43 & 2 & 202.40 & 154.68 \\
\hline & & 2.5 & 9 & 194.63 & 859.31 & 9 & 192.73 & 843.67 & 8 & 192.24 & 818.66 \\
\hline & & 5.0 & 15 & 190.61 & 1541.34 & 15 & 189.16 & 1526.25 & 14 & 188.78 & 1501.17 \\
\hline & & 7.5 & 21 & 186.43 & 2072.38 & 20 & 186.94 & 2059.96 & 19 & 187.18 & 2038.07 \\
\hline & & 10.0 & 26 & 183.90 & 2484.05 & 25 & 184.59 & 2473.96 & 25 & 183.05 & 2456.00 \\
\hline & \multirow{5}{*}{0.05} & 0.5 & 2 & 39.97 & 29.26 & 2 & 37.96 & 24.00 & 1 & 42.39 & 17.55 \\
\hline & & 2.5 & 7 & 38.59 & 152.82 & 6 & 39.03 & 144.89 & 6 & 36.69 & 133.73 \\
\hline & & 5.0 & 12 & 37.93 & 272.59 & 11 & 38.30 & 265.95 & 10 & 38.64 & 255.86 \\
\hline & & 7.5 & 16 & 37.81 & 359.30 & 16 & 37.23 & 354.45 & 15 & 37.46 & 347.25 \\
\hline & & 10.0 & 20 & 37.36 & 420.15 & 20 & 37.00 & 416.90 & 19 & 37.33 & 412.02 \\
\hline & \multirow{5}{*}{0.10} & 0.5 & 2 & 18.92 & 11.63 & 1 & 21.15 & 8.62 & 1 & 18.54 & 5.15 \\
\hline & & 2.5 & 6 & 19.15 & 68.31 & 5 & 19.54 & 63.28 & 5 & 17.96 & 56.97 \\
\hline & & 5.0 & 10 & 19.27 & 120.49 & 10 & 18.68 & 116.75 & 9 & 18.94 & 111.79 \\
\hline & & 7.5 & 14 & 18.85 & 154.85 & 13 & 19.24 & 152.51 & 13 & 18.81 & 149.29 \\
\hline & & 10.0 & 17 & 18.78 & 175.77 & 16 & 19.15 & 174.51 & 16 & 18.94 & 172.72 \\
\hline
\end{tabular}

Dynamic pricing with no acquisition and menu costs $(r=0.1)$.

Proof follows from the fact that, with $r=h=0$, as $\tau \rightarrow \infty$, the optimization problem with $n$ items on hand reduces to $\kappa_{n}^{*}(\infty, y)=\max _{p}-C \mathbf{1}_{(y \neq p)}+p+\kappa_{n-1}^{*}(\infty, p)$; and, that starting with $n=1$ and by induction, the optimal pricing decision is found to be a fixed price independent of $n$.

In the presence of non-zero discount rate and unit holding cost, such an asymptotic result does not hold. Nevertheless, the optimal constant pricing policy can be a reasonable and easily implementable heuristic policy. Hence, we propose the single price policy as Heuristic I. Under this heuristic, only a single price is used throughout the horizon, and the following optimization problem is solved:

$\kappa_{Q}^{I^{*}}\left(\tau_{Q}, 0\right)=\max _{p} \Theta_{n}^{I}\left(\tau_{n}, 0, p\right)$,

where

$\Theta_{n}^{I}\left(\tau_{n}, 0, p\right)=-C \mathbf{1}_{(p \neq 0)}+\sum_{n=1}^{Q} \breve{\Gamma}_{n}\left(\tau_{Q}, p\right)$,

$$
\begin{aligned}
\breve{\Gamma}_{n}\left(\tau_{n}, p\right)= & \int_{0}^{\tau_{Q}} p e^{-r x} d G_{p}^{(n)}(x)-\int_{0}^{\tau_{Q}} h \frac{1-e^{-r x}}{r} d G_{p}^{(n)}(x) \\
& -\left(h \frac{1-e^{-r \tau_{Q}}}{r}+\pi e^{-r \tau_{Q}}\right) \bar{G}_{p}^{(n)}\left(\tau_{Q}\right),
\end{aligned}
$$

and $G_{p}^{(n)}(x)$ denotes the $n$th convolution of $G_{p}(x)$. Heuristic I is appealing in its simplicity and is amenable to obtaining analytical solutions for certain demand distributions. (See Yildirim, 2001.)

The second heuristic we propose, Heuristic II, is a myopic (single-period-look-ahead) policy, under which we use the pricing policy maximizing the within-period profit $\Gamma_{n}\left(\tau_{n}, p\right)$ in any period $n$. That is, Heuristic II uses the optimal prices obtained for the following optimization problem:

$\kappa_{n}^{I I^{*}}\left(\tau_{n}, y\right)=\max _{p} \Theta_{n}^{I I}\left(\tau_{n}, y, p\right)$,

where

$\Theta_{n}^{I I}\left(p, \tau_{n}, y\right)=-C \mathbf{1}_{(y \neq p)}+\Gamma_{n}\left(\tau_{n}, p\right)$. 
Note that $\Theta_{n}^{I I}\left(\tau_{n}, y, p\right)$ is constructed by setting the expected profit-to-go function in Eq. (2) equal to zero from period $n-1$ through period 1 .

By design, Heuristic II ignores the rest of the horizon beyond the current period, say, $n$; thus, it effectively assumes that the remaining $n-1$ items have no contribution. It is an easily computable heuristic, and as such it is appealing; but it may suffer from extreme myopia. We propose two modifications of it by taking into consideration the remaining horizon.

Heuristic III is a modification of the myopic policy which uses the optimal prices obtained for the following optimization problem:

$\kappa_{n}^{I I I^{*}}\left(\tau_{n}, y\right)=\max _{p} \Theta_{n}^{I I I}\left(\tau_{n}, y, p\right)$,

where

$$
\begin{aligned}
\Theta_{n}^{I I I}\left(\tau_{n}, y, p\right)= & -C \mathbf{1}_{(y \neq p)}+\Gamma_{n}\left(\tau_{n}, p\right) \\
& +\int_{0}^{\tau_{n}} \tilde{\kappa}_{n-1}^{*}\left(\tau_{n}-x, p\right) e^{-r x} d G_{p}(x)
\end{aligned}
$$

and $\tilde{\kappa}_{n-1}^{*}\left(\tau_{n}-x, p\right)$ is the optimal profit function as defined in Eq. (2) with menu costs set to zero $(C=0)$ from period $n-1$ to the end of the horizon. The rationale behind this heuristic is to consider the best possible profit-to-go values beyond the current period. It assumes that the remaining $n-1$ items make the maximum possible contribution given the market characteristics (i.e., price sensitivity and demand uncertainty).

Lastly, we propose Heuristic IV-a modification of Heuristic I-which uses the optimal single price policy computed successively for each segment of the remaining horizon from period $n$ to the end. Under this heuristic, at every period $n$ with remaining shelf life $\tau_{n}$, for $1 \leqslant n \leqslant Q$, the optimal single price is obtained by solving the following optimization problem:

$\kappa_{n}^{I V^{*}}\left(\tau_{n}, y\right)=\max _{p} \Theta_{n}^{I V}\left(\tau_{n}, y, p\right)$,

where

$\Theta_{n}^{I V}\left(\tau_{n}, y, p\right)=-C \mathbf{1}_{(y \neq p)}+\sum_{j=1}^{n} \breve{\Gamma}_{j}\left(\tau_{n}, p\right)$,

Table 3

\begin{tabular}{|c|c|c|c|c|c|c|c|c|c|c|c|}
\hline \multirow[t]{2}{*}{$b$} & \multirow[t]{2}{*}{$a$} & \multirow[t]{2}{*}{$\tau$} & \multicolumn{3}{|c|}{$\pi=5$} & \multicolumn{3}{|c|}{$\pi=10$} & \multicolumn{3}{|c|}{$\pi=20$} \\
\hline & & & $Q^{*}$ & $p^{*}$ & $\kappa^{*}$ & $Q^{*}$ & $p^{*}$ & $\kappa^{*}$ & $Q^{*}$ & $p^{*}$ & $\kappa^{*}$ \\
\hline \multirow{15}{*}{3} & \multirow{5}{*}{0.01} & 0.5 & 1 & 173 & 48.13 & 1 & 171 & 45.51 & 1 & 168 & 40.34 \\
\hline & & 2.5 & 5 & 162 & 350.15 & 5 & 160 & 341.43 & 4 & 168 & 329.43 \\
\hline & & 5 & 8 & 165 & 739.23 & 8 & 164 & 730.39 & 8 & 161 & 713.28 \\
\hline & & 7.5 & 12 & 161 & 1120.56 & 12 & 160 & 1109.58 & 11 & 164 & 1089.79 \\
\hline & & 10 & 15 & 162 & 1487.43 & 15 & 161 & 1476.86 & 15 & 160 & 1456.75 \\
\hline & \multirow{5}{*}{0.05} & 0.5 & 1 & 33 & 7.27 & 1 & 31 & 4.78 & 1 & 28 & 0.10 \\
\hline & & 2.5 & 4 & 33 & 59.80 & 4 & 32 & 54.59 & 3 & 33 & 48.69 \\
\hline & & 5 & 7 & 34 & 123.45 & 7 & 33 & 117.63 & 6 & 34 & 109.70 \\
\hline & & 7.5 & 10 & 34 & 180.50 & 9 & 35 & 174.78 & 9 & 34 & 166.64 \\
\hline & & 10 & 12 & 35 & 230.98 & 12 & 34 & 226.13 & 11 & 35 & 218.25 \\
\hline & \multirow{5}{*}{0.10} & 0.5 & 1 & 16 & 2.21 & 0 & - & 0 & 0 & - & 0 \\
\hline & & 2.5 & 3 & 17 & 25.14 & 3 & 16 & 22.36 & 2 & 17 & 18.21 \\
\hline & & 5 & 6 & 17 & 51.03 & 5 & 18 & 47.71 & 5 & 17 & 43.71 \\
\hline & & 7.5 & 8 & 18 & 71.76 & 8 & 17 & 68.79 & 7 & 18 & 65.04 \\
\hline & & 10 & 10 & 18 & 87.71 & 10 & 18 & 85.21 & 9 & 18 & 82.45 \\
\hline \multirow{15}{*}{4} & \multirow{5}{*}{0.01} & 0.5 & 2 & 215 & 114.02 & 2 & 213 & 108.28 & 2 & 209 & 96.90 \\
\hline & & 2.5 & 7 & 209 & 722.07 & 6 & 216 & 710.63 & 6 & 212 & 693.34 \\
\hline & & 5 & 12 & 207 & 1498.94 & 12 & 206 & 1485.08 & 11 & 209 & 1459.69 \\
\hline & & 7.5 & 17 & 204 & 2264.72 & 17 & 203 & 2249.70 & 16 & 205 & 2221.27 \\
\hline & & 10 & 20 & 210 & 2996.44 & 20 & 209 & 2984.59 & 20 & 208 & 2961.88 \\
\hline & \multirow{5}{*}{0.05} & 0.5 & 1 & 47 & 17.83 & 1 & 45 & 15.70 & 1 & 42 & 11.68 \\
\hline & & 2.5 & 6 & 43 & 128.83 & 5 & 44 & 121.73 & 5 & 42 & 111.24 \\
\hline & & 5 & 10 & 44 & 262.57 & 10 & 43 & 254.44 & 9 & 43 & 241.29 \\
\hline & & 7.5 & 15 & 42 & 387.44 & 14 & 43 & 378.96 & 13 & 43 & 365.44 \\
\hline & & 10 & 19 & 42 & 502.17 & 18 & 43 & 494.01 & 18 & 42 & 481.56 \\
\hline & \multirow{5}{*}{0.10} & 0.5 & 1 & 23 & 7.68 & 1 & 21 & 5.68 & 1 & 18 & 2.06 \\
\hline & & 2.5 & 5 & 22 & 57.29 & 5 & 21 & 52.16 & 4 & 21 & 46.22 \\
\hline & & 5 & 9 & 22 & 114.08 & 8 & 23 & 108.53 & 8 & 22 & 101.35 \\
\hline & & 7.5 & 13 & 22 & 162.62 & 12 & 22 & 157.63 & 11 & 23 & 150.73 \\
\hline & & 10 & 16 & 22 & 203.28 & 15 & 23 & 199.17 & 15 & 22 & 193.48 \\
\hline
\end{tabular}

Optimal starting price, initial stock and expected profit.

Dynamic pricing $\left(r=0.01, c_{o}=0.3 / a, C=10\right)$. 
and

$$
\begin{aligned}
\breve{\Gamma}_{j}\left(\tau_{n}, p\right)= & \int_{0}^{\tau_{n}} p e^{-r x} d G_{p}^{(j)}(x)-\int_{0}^{\tau_{n}} h \frac{1-e^{-r x}}{r} d G_{p}^{(j)}(x) \\
& -\left(h \frac{1-e^{-r \tau_{n}}}{r}+\pi e^{-r \tau_{n}}\right) \bar{G}_{p}^{(j)}\left(\tau_{n}\right) .
\end{aligned}
$$

The rationale behind this heuristic is to consider a reasonable lower bound on the possible profit-to-go values beyond the current period. It assumes that the remaining $n-1$ items make some reasonable contribution. Heuristic I may also be viewed as a special case of this heuristic.

Due to their design, we would expect Heuristic III to perform better than Heuristic II, and Heuristic IV better than Heuristic I. This is confirmed by our numerical study as discussed below. The optimal pricing policies for all of the proposed heuristics are, under certain conditions, of the three-region type introduced in Section 3. We state this result formally below.

Corollary 3 (Optimal pricing for the policy heuristics). Suppose the conditions in Corollary 1 hold for $G_{p}^{(n)}(x)$ where
$1 \leqslant n \leqslant Q$; and, furthermore, $d / d p \bar{G}_{p}(x)$ is convex in $p$ when $i=I I I$ (i.e., for Heuristic III). Then, for $i=I, I I, I I I, I V$ :

(a) $\Theta_{n}^{i}\left(\tau_{n}, y, p\right)$ is C-concave in $p$;

(b) For $n>0$, any $\tau_{n}$ and $i=I, I I, I I I, I V$, let $p^{i^{*}}\left(\tau_{n}, n\right)$ be the maximizer of $\Theta_{n}^{i}\left(\tau_{n}, y, p\right)$ and $\Theta_{n}^{i}\left(\tau_{n}, y, p^{* i}\left(\tau_{n}, n, y\right)\right)$ denote the corresponding maximum; $p_{L}^{i}\left(\tau_{n}, n\right)$ and $p_{U}^{i}\left(\tau_{n}, n\right)$ be the prices such that $\Theta_{n}^{i}\left(\tau_{n}, y, p_{L}^{i}\left(\tau_{n}, n\right)\right)=\Theta_{n}^{i}\left(\tau_{n}, y, p_{U}^{i}\left(\tau_{n}, n\right)\right)=\Theta_{n}^{i}\left(\tau_{n}\right.$, $\left.y, p^{* i}\left(\tau_{n}, n, y\right)\right)-C$. It is optimal to raise the selling price to $p^{* i}\left(\tau_{n}, n, y\right)$ if the previous price was set either (at or) below $p_{L}^{i}\left(\tau_{n}, n\right)$ or (at or) above $p_{U}^{i}\left(\tau_{n}, n\right)$; otherwise, it is optimal to keep the price in place.

The results for Heuristic II immediately follow from Corollary 2. For the other heuristics, they follow from the supposed convexity properties of $\bar{G}_{p}(x)$ and $d / d p \bar{G}_{p}(x)$ and their proof is similar to that of Corollary 2 .

Next, we compare the performances of the dynamic pricing policy and the proposed heuristics. With each policy heuristic, we obtained its optimal pricing decisions

Table 4

\begin{tabular}{|c|c|c|c|c|c|c|c|c|c|c|c|}
\hline \multirow[t]{2}{*}{$b$} & \multirow[t]{2}{*}{$a$} & \multirow[t]{2}{*}{$\tau$} & \multicolumn{3}{|c|}{$\pi=5$} & \multicolumn{3}{|c|}{$\pi=10$} & \multicolumn{3}{|c|}{$\pi=20$} \\
\hline & & & $Q^{*}$ & $p^{*}$ & $\kappa^{*}$ & $Q^{*}$ & $p^{*}$ & $\kappa^{*}$ & $Q^{*}$ & $p^{*}$ & $\kappa^{*}$ \\
\hline \multirow{15}{*}{3} & \multirow{5}{*}{0.01} & 0.5 & 1 & 172.67 & 48.13 & 1 & 170.95 & 45.51 & 1 & 167.54 & 40.34 \\
\hline & & 2.5 & 5 & 164.01 & 347.77 & 4 & 172.58 & 339.25 & 4 & 169.94 & 327.71 \\
\hline & & 5.0 & 8 & 170.09 & 729.91 & 8 & 168.85 & 720.67 & 8 & 166.45 & 702.78 \\
\hline & & 7.5 & 12 & 167.47 & 1099.56 & 12 & 166.29 & 1087.76 & 11 & 169.78 & 1067.58 \\
\hline & & 10.0 & 15 & 169.69 & 1452.80 & 15 & 168.68 & 1440.93 & 15 & 166.73 & 1418.08 \\
\hline & \multirow{5}{*}{0.05} & 0.5 & 1 & 33.08 & 7.27 & 1 & 31.42 & 4.78 & 1 & 28.23 & 0.10 \\
\hline & & 2.5 & 4 & 33.37 & 59.57 & 4 & 32.14 & 54.33 & 3 & 33.38 & 48.57 \\
\hline & & 5.0 & 7 & 34.08 & 122.64 & 7 & 33.14 & 116.76 & 6 & 33.74 & 109.10 \\
\hline & & 7.5 & 10 & 34.23 & 178.54 & 9 & 34.97 & 173.24 & 9 & 33.79 & 164.60 \\
\hline & & 10.0 & 12 & 35.37 & 227.80 & 12 & 34.78 & 222.51 & 11 & 35.12 & 214.42 \\
\hline & \multirow{5}{*}{0.10} & 0.5 & 1 & 15.66 & 2.21 & 0 & - & 0 & 0 & - & 0 \\
\hline & & 2.5 & 3 & 17.40 & 25.12 & 3 & 16.50 & 22.35 & 2 & 17.38 & 18.21 \\
\hline & & 5.0 & 6 & 17.23 & 50.78 & 5 & 17.77 & 47.56 & 5 & 16.83 & 43.52 \\
\hline & & 7.5 & 8 & 17.84 & 71.32 & 8 & 17.35 & 68.37 & 7 & 17.58 & 64.66 \\
\hline & & 10.0 & 10 & 18.13 & 86.99 & 9 & 18.50 & 84.79 & 9 & 17.96 & 81.56 \\
\hline \multirow{15}{*}{4} & \multirow{5}{*}{0.01} & 0.5 & 2 & 214.92 & 113.65 & 2 & 212.96 & 107.90 & 2 & 209.04 & 96.51 \\
\hline & & 2.5 & 7 & 213.87 & 710.86 & 6 & 221.67 & 700.96 & 6 & 218.79 & 683.14 \\
\hline & & 5.0 & 12 & 216.56 & 1460.11 & 12 & 215.14 & 1445.29 & 11 & 219.34 & 1420.99 \\
\hline & & 7.5 & 17 & 216.97 & 2183.69 & 16 & 220.87 & 2167.56 & 16 & 218.63 & 2139.20 \\
\hline & & 10.0 & 20 & 225.37 & 2872.11 & 20 & 224.39 & 2857.52 & 20 & 222.48 & 2829.23 \\
\hline & \multirow{5}{*}{0.05} & 0.5 & 1 & 46.67 & 17.83 & 1 & 45.18 & 15.70 & 1 & 42.31 & 11.68 \\
\hline & & 2.5 & 6 & 43.11 & 127.42 & 5 & 44.54 & 120.78 & 5 & 42.43 & 110.19 \\
\hline & & 5.0 & 10 & 44.64 & 257.78 & 10 & 43.65 & 249.24 & 9 & 44.03 & 236.52 \\
\hline & & 7.5 & 14 & 45.21 & 376.17 & 14 & 44.41 & 367.40 & 13 & 44.62 & 353.75 \\
\hline & & 10.0 & 18 & 45.45 & 482.77 & 17 & 45.98 & 474.08 & 17 & 44.88 & 460.47 \\
\hline & \multirow{5}{*}{0.10} & 0.5 & 1 & 22.54 & 7.68 & 1 & 21.11 & 5.68 & 1 & 18.44 & 2.06 \\
\hline & & 2.5 & 5 & 22.07 & 56.80 & 4 & 22.87 & 51.75 & 4 & 21.38 & 45.92 \\
\hline & & 5.0 & 9 & 22.46 & 112.49 & 8 & 22.86 & 107.26 & 8 & 21.75 & 99.85 \\
\hline & & 7.5 & 12 & 23.30 & 159.40 & 12 & 22.74 & 154.47 & 11 & 22.82 & 147.40 \\
\hline & & 10.0 & 15 & 23.74 & 197.94 & 15 & 23.31 & 193.62 & 14 & 23.38 & 187.27 \\
\hline
\end{tabular}

Optimal starting price, initial stock and expected profit.

Heuristic I $\left(r=0.01, c_{o}=0.3 / a, C=10\right)$. 
at any system state given by $\left(n, \tau_{n}, y\right)$ as defined before. Using these prices, we then evaluated the corresponding expected discounted profit in the presence of menu costs; and, searched for the best system profit over the initial stocking level $Q$, with given acquisition costs. We denote this value by $\kappa_{Q^{*}}\left(\tau, p_{Q^{*}}^{* i}\right)$ for Heuristic $i,(i=I, I I, I I I, I V)$. We define $\Delta \kappa^{*}$ to be the relative optimal discounted expected profit improvement as follows:

$\Delta \kappa^{*}=\frac{\kappa_{Q^{*}}\left(\tau, p_{Q^{*}}^{*^{d}}\right)-\kappa_{Q^{*}}\left(\tau, p_{Q^{*}}^{*^{i}}\right)}{\kappa_{Q^{*}}\left(\tau, p_{Q^{*}}^{*^{d}}\right)} \times 100$,

where superscript $d$ refers to dynamic pricing and $i$ refers to Heuristic $i$. $i=I, I I, I I I, I V$. Note that we allow for different optimal initial stocking levels in our comparisons in the presence of non-negligible acquisition costs $(v(Q)>0)$.

In Tables 3-7, we present illustrative examples of the optimal stocking levels, starting prices and the corresponding expected profits under dynamic pricing and the proposed heuristics. The best initial stocking levels, $Q^{*}$ under all four heuristics are never larger than the optimal;

Table 5

Optimal starting price, initial stock and expected profit.

\begin{tabular}{|c|c|c|c|c|c|c|c|c|c|c|}
\hline \multirow[t]{2}{*}{$a$} & \multirow[t]{2}{*}{$\tau$} & \multicolumn{3}{|c|}{$\pi=5$} & \multicolumn{3}{|c|}{$\pi=10$} & \multicolumn{3}{|c|}{$\pi=20$} \\
\hline & & $Q^{*}$ & $p^{*}$ & $\kappa^{*}$ & $Q^{*}$ & $p^{*}$ & $\kappa^{*}$ & $Q^{*}$ & $p^{*}$ & $\kappa^{*}$ \\
\hline \multirow{5}{*}{0.01} & 0.5 & 1 & 173 & 48.13 & 1 & 171 & 45.51 & 1 & 168 & 40.34 \\
\hline & 2.5 & 3 & 221 & 285.16 & 3 & 219 & 283.70 & 3 & 216 & 278.66 \\
\hline & 5.0 & 5 & 243 & 501.58 & 5 & 242 & 501.48 & 5 & 239 & 504.94 \\
\hline & 7.5 & 6 & 254 & 662.27 & 6 & 253 & 667.67 & 6 & 251 & 675.91 \\
\hline & 10.0 & 8 & 258 & 812.04 & 8 & 258 & 811.04 & 8 & 256 & 829.95 \\
\hline \multirow{5}{*}{0.05} & 0.5 & 1 & 33 & 7.27 & 1 & 31 & 4.78 & 1 & 28 & 0.10 \\
\hline & 2.5 & 3 & 42 & 52.75 & 3 & 41 & 49.53 & 3 & 38 & 45.66 \\
\hline & 5.0 & 5 & 46 & 91.70 & 5 & 45 & 90.22 & 4 & 45 & 85.09 \\
\hline & 7.5 & 6 & 47 & 131.33 & 7 & 46 & 126.37 & 6 & 46 & 123.84 \\
\hline & 10.0 & 10 & 45 & 176.27 & 7 & 47 & 166.37 & 8 & 46 & 162.41 \\
\hline \multirow{5}{*}{0.10} & 0.5 & 1 & 16 & 2.21 & 0 & - & 0 & 0 & - & 0 \\
\hline & 2.5 & 3 & 20 & 23.27 & 3 & 19 & 20.65 & 2 & 19 & 17.64 \\
\hline & 5.0 & 5 & 21 & 45.31 & 5 & 21 & 39.98 & 4 & 21 & 37.21 \\
\hline & 7.5 & 7 & 21 & 64.71 & 7 & 21 & 59.29 & 6 & 21 & 56.94 \\
\hline & 10.0 & 9 & 20 & 85.20 & 9 & 20 & 81.56 & 7 & 21 & 75.78 \\
\hline \multirow{5}{*}{0.01} & 0.5 & 2 & 238 & 111.33 & 2 & 235 & 105.82 & 2 & 229 & 94.89 \\
\hline & 2.5 & 4 & 309 & 538.95 & 4 & 307 & 538.36 & 4 & 304 & 533.97 \\
\hline & 5.0 & 7 & 336 & 897.58 & 7 & 335 & 900.78 & 7 & 332 & 913.37 \\
\hline & 7.5 & 8 & 349 & 1167.26 & 8 & 348 & 1179.91 & 8 & 346 & 1200.07 \\
\hline & 10.0 & 10 & 354 & 1406.64 & 9 & 354 & 1422.88 & 10 & 352 & 1448.54 \\
\hline \multirow{5}{*}{$\begin{array}{ll}4 & 0.05\end{array}$} & 0.5 & 1 & 47 & 17.83 & 1 & 45 & 15.70 & 1 & 42 & 11.68 \\
\hline & 2.5 & 4 & 60 & 99.27 & 4 & 58 & 99.11 & 4 & 56 & 92.73 \\
\hline & 5.0 & 5 & 65 & 164.88 & 5 & 64 & 167.47 & 5 & 63 & 166.22 \\
\hline & 7.5 & 9 & 65 & 234.31 & 8 & 65 & 236.52 & 7 & 65 & 232.21 \\
\hline & 10.0 & 13 & 63 & 324.51 & 13 & 63 & 311.79 & 11 & 64 & 304.37 \\
\hline \multirow{5}{*}{0.10} & 0.5 & 1 & 23 & 7.68 & 1 & 21 & 5.68 & 1 & 18 & 2.06 \\
\hline & 2.5 & 3 & 29 & 46.41 & 3 & 28 & 44.86 & 3 & 27 & 40.81 \\
\hline & 5.0 & 7 & 30 & 85.32 & 6 & 30 & 82.58 & 5 & 30 & 77.17 \\
\hline & 7.5 & 9 & 30 & 127.42 & 9 & 30 & 118.25 & 8 & 30 & 114.40 \\
\hline & 10.0 & 11 & 29 & 175.67 & 11 & 29 & 169.70 & 11 & 29 & 159.82 \\
\hline
\end{tabular}

Heuristic II $\left(r=0.01, c_{o}=0.3 / a, C=10\right)$.
Heuristic II results in significantly lower levels of initial stock. The impact of menu cost and discount rate on $Q^{*}$ is not discernable. The best starting prices, $p^{*}$ are, typically, lower under Heuristics I, II and IV, and are higher under Heuristic II than the prices under the dynamic pricing policy. Heuristic IV results in the optimal starting prices for many of the experiments. The deviation from the optimal starting price decreases (respectively, increases) for heuristics I, II, III (respectively, IV) as menu cost and discount rate increase. The results on $p^{*}$ and $Q^{*}$ complement each other: as the pricing decision gets more myopic, the system tends to compensate for this aggressive behavior by holding less stock hedging against excess unsold stocks.

Tables 8-19 provide comparisons of the heuristics with respect to optimal solutions in terms of $\Delta \kappa^{*}$. The oneperiod-look-ahead policy, Heuristic II, has the worst performance among the tested heuristic policies. As expected, it worsens with lower discount rates and improves with higher menu costs. Its overall performance tends to improve as price sensitivity $a$ increases and base demand rate $b$ decreases. $\Delta \kappa^{*}$ is initially increasing but

Table 6

Optimal starting price, initial stock and expected profit.

\begin{tabular}{|c|c|c|c|c|c|c|c|c|c|c|c|}
\hline \multirow[t]{2}{*}{$b$} & \multirow[t]{2}{*}{$a$} & \multirow[t]{2}{*}{$\tau$} & \multicolumn{3}{|c|}{$\pi=5$} & \multicolumn{3}{|c|}{$\pi=10$} & \multicolumn{3}{|c|}{$\pi=20$} \\
\hline & & & $Q^{*}$ & $p^{*}$ & $\kappa^{*}$ & $Q^{*}$ & $p^{*}$ & $\kappa^{*}$ & $Q^{*}$ & $p^{*}$ & $\kappa^{*}$ \\
\hline \multirow{5}{*}{\multicolumn{2}{|c|}{0.01}} & 0.5 & 1 & 173 & 48.13 & 1 & 171 & 45.51 & 1 & 168 & 40.34 \\
\hline & & 2.5 & 5 & 157 & 349.35 & 5 & 155 & 340.54 & 4 & 161 & 328.29 \\
\hline & & 5.0 & 8 & 161 & 737.22 & 8 & 160 & 728.51 & 8 & 157 & 711.35 \\
\hline & & 7.5 & 12 & 158 & 1117.52 & 12 & 156 & 1106.13 & 11 & 160 & 1086.54 \\
\hline & & 10.0 & 15 & 159 & 1482.44 & 15 & 158 & 1472.00 & 15 & 156 & 1451.66 \\
\hline \multirow{5}{*}{3} & \multirow{5}{*}{0.05} & 0.5 & 1 & 33 & 7.27 & 1 & 31 & 4.78 & 1 & 28 & 0.10 \\
\hline & & 2.5 & 4 & 31 & 59.10 & 4 & 30 & 54.00 & 3 & 31 & 48.04 \\
\hline & & 5.0 & 7 & 32 & 122.22 & 7 & 31 & 116.28 & 6 & 32 & 108.85 \\
\hline & & 7.5 & 9 & 33 & 176.32 & 9 & 33 & 172.81 & 9 & 32 & 164.58 \\
\hline & & 10.0 & 11 & 34 & 224.14 & 12 & 32 & 220.26 & 11 & 33 & 213.65 \\
\hline \multirow{5}{*}{\multicolumn{2}{|c|}{0.10}} & 0.5 & 1 & 16 & 2.21 & 0 & - & 0 & 0 & - & 0 \\
\hline & & 2.5 & 3 & 16 & 24.73 & 3 & 15 & 21.90 & 2 & 17 & 18.21 \\
\hline & & 5.0 & 6 & 16 & 50.22 & 5 & 17 & 47.43 & 5 & 16 & 43.34 \\
\hline & & 7.5 & 8 & 16 & 68.94 & 7 & 17 & 67.30 & 7 & 17 & 64.75 \\
\hline & & 10.0 & 9 & 17 & 83.71 & 9 & 17 & 82.82 & 9 & 17 & 81.05 \\
\hline \multirow{5}{*}{\multicolumn{2}{|c|}{0.01}} & 0.5 & 2 & 209 & 113.88 & 2 & 207 & 108.13 & 2 & 202 & 96.69 \\
\hline & & 2.5 & 7 & 203 & 720.87 & 6 & 210 & 709.37 & 6 & 207 & 692.09 \\
\hline & & 5.0 & 12 & 202 & 1495.60 & 12 & 200 & 1481.64 & 11 & 204 & 1456.69 \\
\hline & & 7.5 & 17 & 198 & 2257.38 & 17 & 196 & 2241.90 & 16 & 200 & 2214.79 \\
\hline & & 10.0 & 20 & 204 & 2985.48 & 20 & 203 & 2973.64 & 20 & 201 & 2950.86 \\
\hline \multirow{5}{*}{\multicolumn{2}{|c|}{40.05}} & 0.5 & 1 & 47 & 17.83 & 1 & 45 & 15.70 & 1 & 42 & 11.68 \\
\hline & & 2.5 & 6 & 40 & 127.63 & 5 & 42 & 120.94 & 5 & 39 & 109.74 \\
\hline & & 5.0 & 10 & 41 & 258.87 & 10 & 40 & 250.69 & 9 & 41 & 238.23 \\
\hline & & 7.5 & 14 & 41 & 379.36 & 14 & 40 & 370.91 & 13 & 41 & 358.58 \\
\hline & & 10.0 & 19 & 39 & 488.18 & 18 & 40 & 481.88 & 18 & 39 & 469.25 \\
\hline \multirow{5}{*}{\multicolumn{2}{|c|}{0.10}} & 0.5 & 1 & 23 & 7.68 & 1 & 21 & 5.68 & 1 & 18 & 2.06 \\
\hline & & 2.5 & 5 & 21 & 57.00 & 4 & 21 & 51.17 & 4 & 20 & 45.77 \\
\hline & & 5.0 & 8 & 22 & 111.64 & 9 & 20 & 106.80 & 7 & 22 & 99.67 \\
\hline & & 7.5 & 12 & 21 & 157.34 & 12 & 21 & 154.55 & 11 & 21 & 146.64 \\
\hline & & 10.0 & 14 & 22 & 193.37 & 14 & 22 & 191.79 & 14 & 21 & 183.63 \\
\hline
\end{tabular}

Heuristic III $\left(r=0.01, c_{o}=0.3 / a, C=10\right)$. 
Table 7

Optimal starting price, initial stock and expected profit.

\begin{tabular}{|c|c|c|c|c|c|c|c|c|c|c|c|}
\hline \multirow[t]{2}{*}{$b$} & \multirow[t]{2}{*}{$a$} & \multirow[t]{2}{*}{$\tau$} & \multicolumn{3}{|c|}{$\pi=5$} & \multicolumn{3}{|c|}{$\pi=10$} & \multicolumn{3}{|c|}{$\pi=20$} \\
\hline & & & $Q^{*}$ & $p^{*}$ & $\kappa^{*}$ & $Q^{*}$ & $p^{*}$ & $\kappa^{*}$ & $Q^{*}$ & $p^{*}$ & $\kappa^{*}$ \\
\hline \multirow{15}{*}{3} & \multirow{5}{*}{0.01} & 0.5 & 1 & 173 & 48.13 & 1 & 171 & 45.51 & 1 & 168 & 40.34 \\
\hline & & 2.5 & 5 & 164 & 350.02 & 5 & 162 & 341.27 & 4 & 170 & 329.31 \\
\hline & & 5.0 & 8 & 169 & 737.32 & 8 & 168 & 728.27 & 8 & 166 & 710.86 \\
\hline & & 7.5 & 12 & 167 & 1115.57 & 12 & 165 & 1104.44 & 11 & 169 & 1085.06 \\
\hline & & 10.0 & 15 & 169 & 1479.99 & 15 & 168 & 1469.40 & 15 & 166 & 1448.95 \\
\hline & \multirow{5}{*}{0.05} & 0.5 & 1 & 33 & 7.27 & 1 & 31 & 4.78 & 1 & 28 & 0.10 \\
\hline & & 2.5 & 4 & 33 & 59.80 & 4 & 32 & 54.59 & 3 & 33 & 48.69 \\
\hline & & 5.0 & 7 & 34 & 123.45 & 7 & 33 & 117.63 & 6 & 34 & 109.70 \\
\hline & & 7.5 & 10 & 34 & 180.49 & 9 & 35 & 174.78 & 9 & 34 & 166.61 \\
\hline & & 10.0 & 12 & 35 & 230.86 & 12 & 35 & 225.66 & 11 & 35 & 218.09 \\
\hline & \multirow{5}{*}{0.10} & 0.5 & 1 & 16 & 2.21 & 0 & - & 0 & 0 & - & 0 \\
\hline & & 2.5 & 3 & 17 & 25.14 & 3 & 16 & 22.36 & 2 & 17 & 18.21 \\
\hline & & 5.0 & 6 & 17 & 51.03 & 5 & 18 & 47.71 & 5 & 17 & 43.71 \\
\hline & & 7.5 & 8 & 18 & 71.76 & 8 & 17 & 68.79 & 7 & 18 & 65.04 \\
\hline & & 10.0 & 10 & 18 & 87.71 & 10 & 18 & 85.21 & 9 & 18 & 82.45 \\
\hline \multirow{15}{*}{4} & \multirow{5}{*}{0.01} & 0.5 & 2 & 215 & 114.02 & 2 & 213 & 108.28 & 2 & 209 & 96.90 \\
\hline & & 2.5 & 7 & 212 & 721.24 & 6 & 220 & 709.44 & 6 & 218 & 691.82 \\
\hline & & 5.0 & 12 & 214 & 1493.28 & 12 & 213 & 1478.90 & 11 & 217 & 1452.98 \\
\hline & & 7.5 & 17 & 213 & 2253.35 & 17 & 212 & 2237.84 & 16 & 216 & 2208.68 \\
\hline & & 10.0 & 20 & 222 & 2978.09 & 20 & 221 & 2965.50 & 20 & 219 & 2942.75 \\
\hline & \multirow{5}{*}{0.05} & 0.5 & 1 & 47 & 17.83 & 1 & 45 & 15.70 & 1 & 42 & 11.68 \\
\hline & & 2.5 & 6 & 43 & 128.83 & 5 & 44 & 121.73 & 5 & 42 & 111.24 \\
\hline & & 5.0 & 10 & 44 & 262.44 & 10 & 43 & 254.25 & 9 & 44 & 240.94 \\
\hline & & 7.5 & 15 & 43 & 386.45 & 14 & 44 & 377.71 & 13 & 44 & 364.40 \\
\hline & & 10.0 & 19 & 44 & 499.43 & 18 & 44 & 491.78 & 17 & 44 & 479.27 \\
\hline & \multirow{5}{*}{0.10} & 0.5 & 1 & 23 & 7.68 & 1 & 21 & 5.68 & 1 & 18 & 2.06 \\
\hline & & 2.5 & 5 & 22 & 57.29 & 5 & 21 & 52.16 & 4 & 21 & 46.22 \\
\hline & & 5.0 & 9 & 22 & 114.08 & 8 & 23 & 108.53 & 8 & 22 & 101.35 \\
\hline & & 7.5 & 13 & 22 & 162.60 & 12 & 23 & 157.51 & 11 & 23 & 150.68 \\
\hline & & 10.0 & 16 & 23 & 203.12 & 15 & 23 & 199.10 & 15 & 22 & 193.23 \\
\hline
\end{tabular}

Heuristic IV $\left(r=0.01, c_{o}=0.3 / a, C=10\right)$.

Table 8

Relative profit improvement, $\left(\Delta \kappa^{*}\right)$, of dynamic pricing over Heuristic I $\left(r=0.01, c_{o}=0.3 / a, C=5\right)$.

\begin{tabular}{|c|c|c|c|c|c|c|c|}
\hline \multirow[t]{2}{*}{$a$} & \multirow[t]{2}{*}{$\tau$} & \multicolumn{3}{|l|}{$b=3$} & \multicolumn{3}{|l|}{$b=4$} \\
\hline & & $\pi=5$ & $\pi=10$ & $\pi=20$ & $\pi=5$ & $\pi=10$ & $\pi=20$ \\
\hline \multirow{5}{*}{0.01} & 0.5 & 0.00 & 0.00 & 0.00 & 0.32 & 0.35 & 0.40 \\
\hline & 2.5 & 0.89 & 0.86 & 0.84 & 1.70 & 1.58 & 1.71 \\
\hline & 5.0 & 1.53 & 1.61 & 1.79 & 2.75 & 2.85 & 2.89 \\
\hline & 7.5 & 2.12 & 2.23 & 2.42 & 3.74 & 3.83 & 3.93 \\
\hline & 10.0 & 2.60 & 2.73 & 2.99 & 4.36 & 4.48 & 4.72 \\
\hline \multirow{5}{*}{0.05} & 0.5 & 0.00 & 0.00 & 0.00 & 0.00 & 0.00 & 0.00 \\
\hline & 2.5 & 0.38 & 0.48 & 0.25 & 1.21 & 0.94 & 1.22 \\
\hline & 5.0 & 0.88 & 1.09 & 1.11 & 2.27 & 2.58 & 2.79 \\
\hline & 7.5 & 1.69 & 1.65 & 2.40 & 3.40 & 3.69 & 4.10 \\
\hline & 10.0 & 2.16 & 2.54 & 2.95 & 4.43 & 4.74 & 5.26 \\
\hline \multirow{5}{*}{0.10} & 0.5 & 0.00 & - & - & 0.00 & 0.00 & 0.00 \\
\hline & 2.5 & 0.08 & 0.04 & 0.00 & 0.86 & 0.79 & 0.65 \\
\hline & 5.0 & 0.49 & 0.31 & 0.82 & 1.78 & 1.72 & 2.45 \\
\hline & 7.5 & 0.64 & 0.87 & 1.42 & 2.72 & 3.11 & 3.66 \\
\hline & 10.0 & 1.11 & 1.03 & 1.88 & 3.75 & 3.99 & 4.81 \\
\hline
\end{tabular}


Table 9

Relative profit improvement, $\left(\Delta \kappa^{*}\right)$, of dynamic pricing over Heuristic II $\left(r=0.01, c_{o}=0.3 / a, C=5\right)$.

\begin{tabular}{|c|c|c|c|c|c|c|c|}
\hline \multirow[t]{2}{*}{$a$} & \multirow[t]{2}{*}{$\tau$} & \multicolumn{3}{|l|}{$b=3$} & \multicolumn{3}{|l|}{$b=4$} \\
\hline & & $\pi=5$ & $\pi=10$ & $\pi=20$ & $\pi=5$ & $\pi=10$ & $\pi=20$ \\
\hline \multirow{5}{*}{0.01} & 0.5 & 0.00 & 0.00 & 0.00 & 2.36 & 2.27 & 2.07 \\
\hline & 2.5 & 17.64 & 16.19 & 14.81 & 24.32 & 23.02 & 21.66 \\
\hline & 5.0 & 30.64 & 29.82 & 27.80 & 38.50 & 37.70 & 35.90 \\
\hline & 7.5 & 39.05 & 38.04 & 36.22 & 46.94 & 46.11 & 44.54 \\
\hline & 10.0 & 43.71 & 43.31 & 41.34 & 51.58 & 50.98 & 49.65 \\
\hline \multirow{5}{*}{0.05} & 0.5 & 0.00 & 0.00 & 0.00 & 0.00 & 0.00 & 0.00 \\
\hline & 2.5 & 11.79 & 9.27 & 6.22 & 22.83 & 18.32 & 15.43 \\
\hline & 5.0 & 25.46 & 22.15 & 21.01 & 34.49 & 31.87 & 29.79 \\
\hline & 7.5 & 26.96 & 25.96 & 24.05 & 37.32 & 35.72 & 34.88 \\
\hline & 10.0 & 23.73 & 25.87 & 24.12 & 34.15 & 35.34 & 35.01 \\
\hline \multirow{5}{*}{0.10} & 0.5 & 0.00 & - & - & 0.00 & 0.00 & 0.00 \\
\hline & 2.5 & 7.44 & 7.65 & 3.13 & 18.99 & 14.00 & 11.38 \\
\hline & 5.0 & 11.21 & 15.91 & 13.79 & 25.24 & 22.98 & 22.63 \\
\hline & 7.5 & 9.86 & 13.51 & 11.48 & 22.01 & 24.07 & 22.82 \\
\hline & 10.0 & 3.16 & 4.67 & 8.07 & 14.55 & 15.34 & 17.23 \\
\hline
\end{tabular}

Table 10

Relative profit improvement, $\left(\Delta \kappa^{*}\right)$, of dynamic pricing over Heuristic III $\left(r=0.01, c_{o}=0.3 / a, C=5\right)$.

\begin{tabular}{|c|c|c|c|c|c|c|c|}
\hline \multirow[t]{2}{*}{$a$} & \multirow[t]{2}{*}{$\tau$} & \multicolumn{3}{|l|}{$b=3$} & \multicolumn{3}{|l|}{$b=4$} \\
\hline & & $\pi=5$ & $\pi=10$ & $\pi=20$ & $\pi=5$ & $\pi=10$ & $\pi=20$ \\
\hline \multirow{5}{*}{0.01} & 0.5 & 0.00 & 0.00 & 0.00 & 0.12 & 0.14 & 0.22 \\
\hline & 2.5 & 0.13 & 0.13 & 0.14 & 0.10 & 0.08 & 0.08 \\
\hline & 5.0 & 0.12 & 0.11 & 0.12 & 0.10 & 0.11 & 0.10 \\
\hline & 7.5 & 0.12 & 0.15 & 0.19 & 0.16 & 0.17 & 0.14 \\
\hline & 10.0 & 0.17 & 0.18 & 0.21 & 0.17 & 0.18 & 0.19 \\
\hline \multirow{5}{*}{0.05} & 0.5 & 0.00 & 0.00 & 0.00 & 0.00 & 0.00 & 0.00 \\
\hline & 2.5 & 1.14 & 1.03 & 1.33 & 0.74 & 0.51 & 0.93 \\
\hline & 5.0 & 0.84 & 0.95 & 0.85 & 0.86 & 0.86 & 0.81 \\
\hline & 7.5 & 1.88 & 1.03 & 1.27 & 1.16 & 1.17 & 1.13 \\
\hline & 10.0 & 2.04 & 1.67 & 1.64 & 1.55 & 1.35 & 1.43 \\
\hline \multirow{5}{*}{0.10} & 0.5 & 0.00 & - & - & 0.00 & 0.00 & 0.00 \\
\hline & 2.5 & 1.63 & 2.06 & 0.00 & 0.51 & 1.90 & 0.97 \\
\hline & 5.0 & 1.59 & 0.59 & 1.23 & 2.36 & 1.67 & 2.31 \\
\hline & 7.5 & 3.87 & 2.42 & 1.07 & 2.90 & 2.14 & 2.90 \\
\hline & 10.0 & 4.77 & 3.28 & 2.29 & 4.00 & 3.22 & 4.26 \\
\hline
\end{tabular}

Table 11

Relative profit improvement, $\left(\Delta \kappa^{*}\right)$, of dynamic pricing over Heuristic IV $\left(r=0.01, c_{o}=0.3 / a, C=5\right)$.

\begin{tabular}{|c|c|c|c|c|c|c|c|}
\hline \multirow[t]{2}{*}{$a$} & \multirow[t]{2}{*}{$\tau$} & \multicolumn{3}{|l|}{$b=3$} & \multicolumn{3}{|l|}{$b=4$} \\
\hline & & $\pi=5$ & $\pi=10$ & $\pi=20$ & $\pi=5$ & $\pi=10$ & $\pi=20$ \\
\hline \multirow{5}{*}{0.01} & 0.5 & 0.00 & 0.00 & 0.00 & 0.00 & 0.00 & 0.00 \\
\hline & 2.5 & 0.14 & 0.13 & 0.15 & 0.23 & 0.26 & 0.32 \\
\hline & 5.0 & 0.33 & 0.34 & 0.38 & 0.41 & 0.43 & 0.43 \\
\hline & 7.5 & 0.44 & 0.45 & 0.45 & 0.52 & 0.53 & 0.56 \\
\hline & 10.0 & 0.51 & 0.52 & 0.56 & 0.62 & 0.63 & 0.67 \\
\hline \multirow{5}{*}{0.05} & 0.5 & 0.00 & 0.00 & 0.00 & 0.00 & 0.00 & 0.00 \\
\hline & 2.5 & 0.00 & 0.00 & 0.00 & 0.02 & 0.00 & 0.03 \\
\hline & 5.0 & 0.03 & 0.08 & 0.05 & 0.30 & 0.35 & 0.33 \\
\hline & 7.5 & 0.28 & 0.22 & 0.26 & 0.53 & 0.52 & 0.50 \\
\hline & 10.0 & 0.35 & 0.44 & 0.33 & 0.76 & 0.66 & 0.72 \\
\hline
\end{tabular}


Table 11 (continued)

\begin{tabular}{|c|c|c|c|c|c|c|c|}
\hline \multirow[t]{2}{*}{$a$} & \multirow[t]{2}{*}{$\tau$} & \multicolumn{3}{|l|}{$b=3$} & \multicolumn{3}{|l|}{$b=4$} \\
\hline & & $\pi=5$ & $\pi=10$ & $\pi=20$ & $\pi=5$ & $\pi=10$ & $\pi=20$ \\
\hline \multirow{5}{*}{0.10} & 0.5 & 0.00 & - & - & 0.00 & 0.00 & 0.00 \\
\hline & 2.5 & 0.00 & 0.00 & 0.00 & 0.00 & 0.00 & 0.00 \\
\hline & 5.0 & 0.00 & 0.00 & 0.00 & 0.03 & 0.24 & 0.03 \\
\hline & 7.5 & 0.00 & 0.00 & 0.09 & 0.18 & 0.37 & 0.25 \\
\hline & 10.0 & 0.00 & 0.00 & 0.07 & 0.54 & 0.32 & 0.44 \\
\hline
\end{tabular}

Table 12

Relative profit improvement, $\left(\Delta \kappa^{*}\right)$, of dynamic pricing over Heuristic I $\left(r=0.01, c_{o}=0.3 / a, C=10\right)$.

\begin{tabular}{|c|c|c|c|c|c|c|c|}
\hline \multirow[t]{2}{*}{$a$} & \multirow[t]{2}{*}{$\tau$} & \multicolumn{3}{|l|}{$b=3$} & \multicolumn{3}{|l|}{$b=4$} \\
\hline & & $\pi=5$ & $\pi=10$ & $\pi=20$ & $\pi=5$ & $\pi=10$ & $\pi=20$ \\
\hline \multirow{5}{*}{0.01} & 0.5 & 0.00 & 0.00 & 0.00 & 0.32 & 0.35 & 0.40 \\
\hline & 2.5 & 0.68 & 0.64 & 0.52 & 1.55 & 1.36 & 1.47 \\
\hline & 5.0 & 1.26 & 1.33 & 1.47 & 2.59 & 2.68 & 2.65 \\
\hline & 7.5 & 1.87 & 1.97 & 2.04 & 3.58 & 3.65 & 3.69 \\
\hline & 10.0 & 2.33 & 2.43 & 2.65 & 4.15 & 4.26 & 4.48 \\
\hline \multirow{5}{*}{0.05} & 0.5 & 0.00 & 0.00 & 0.00 & 0.00 & 0.00 & 0.00 \\
\hline & 2.5 & 0.38 & 0.48 & 0.25 & 1.09 & 0.78 & 0.94 \\
\hline & 5.0 & 0.66 & 0.74 & 0.55 & 1.82 & 2.04 & 1.98 \\
\hline & 7.5 & 1.09 & 0.88 & 1.22 & 2.91 & 3.05 & 3.20 \\
\hline & 10.0 & 1.38 & 1.60 & 1.75 & 3.86 & 4.03 & 4.38 \\
\hline \multirow{5}{*}{0.10} & 0.5 & 0.00 & - & - & 0.00 & 0.00 & 0.00 \\
\hline & 2.5 & 0.08 & 0.04 & 0.00 & 0.86 & 0.79 & 0.65 \\
\hline & 5.0 & 0.49 & 0.31 & 0.43 & 1.39 & 1.17 & 1.48 \\
\hline & 7.5 & 0.61 & 0.61 & 0.58 & 1.98 & 2.00 & 2.21 \\
\hline & 10.0 & 0.82 & 0.49 & 1.08 & 2.63 & 2.79 & 3.21 \\
\hline
\end{tabular}

Table 13

Relative profit improvement, $\left(\Delta \kappa^{*}\right)$, of dynamic pricing over Heuristic II $\left(r=0.01, c_{o}=0.3 / a, C=10\right)$.

\begin{tabular}{|c|c|c|c|c|c|c|c|}
\hline \multirow[t]{2}{*}{$a$} & \multirow[t]{2}{*}{$\tau$} & \multicolumn{3}{|l|}{$b=3$} & \multicolumn{3}{|l|}{$b=4$} \\
\hline & & $\pi=5$ & $\pi=10$ & $\pi=20$ & $\pi=5$ & $\pi=10$ & $\pi=20$ \\
\hline \multirow{5}{*}{0.01} & 0.5 & 0.00 & 0.00 & 0.00 & 2.36 & 2.27 & 2.07 \\
\hline & 2.5 & 18.56 & 16.91 & 15.41 & 25.36 & 24.24 & 22.99 \\
\hline & 5.0 & 32.15 & 31.34 & 29.21 & 40.12 & 39.34 & 37.43 \\
\hline & 7.5 & 40.90 & 39.83 & 37.98 & 48.46 & 47.55 & 45.97 \\
\hline & 10.0 & 45.41 & 45.08 & 43.03 & 53.06 & 52.33 & 51.09 \\
\hline \multirow{5}{*}{0.05} & 0.5 & 0.00 & 0.00 & 0.00 & 0.00 & 0.00 & 0.00 \\
\hline & 2.5 & 11.79 & 9.27 & 6.22 & 22.94 & 18.58 & 16.64 \\
\hline & 5.0 & 25.72 & 23.30 & 22.43 & 37.21 & 34.18 & 31.11 \\
\hline & 7.5 & 27.24 & 27.70 & 25.68 & 39.52 & 37.59 & 36.46 \\
\hline & 10.0 & 23.69 & 26.43 & 25.59 & 35.38 & 36.89 & 36.79 \\
\hline \multirow{5}{*}{0.10} & 0.5 & 0.00 & - & - & 0.00 & 0.00 & 0.00 \\
\hline & 2.5 & 7.44 & 7.65 & 3.13 & 18.99 & 14.00 & 11.70 \\
\hline & 5.0 & 11.21 & 16.20 & 14.87 & 25.21 & 23.91 & 23.86 \\
\hline & 7.5 & 9.82 & 13.81 & 12.45 & 21.65 & 24.98 & 24.10 \\
\hline & 10.0 & 2.86 & 4.28 & 8.09 & 13.58 & 14.80 & 17.40 \\
\hline
\end{tabular}


Table 14

Relative profit improvement, $\left(\Delta \kappa^{*}\right)$, of dynamic pricing over Heuristic III $\left(r=0.01, c_{o}=0.3 / a, C=10\right)$.

\begin{tabular}{|c|c|c|c|c|c|c|c|}
\hline \multirow[t]{2}{*}{$a$} & \multirow[t]{2}{*}{$\tau$} & \multicolumn{3}{|l|}{$b=3$} & \multicolumn{3}{|l|}{$b=4$} \\
\hline & & $\pi=5$ & $\pi=10$ & $\pi=20$ & $\pi=5$ & $\pi=10$ & $\pi=20$ \\
\hline \multirow{5}{*}{0.01} & 0.5 & 0.00 & 0.00 & 0.00 & 0.12 & 0.14 & 0.22 \\
\hline & 2.5 & 0.23 & 0.26 & 0.35 & 0.17 & 0.18 & 0.18 \\
\hline & 5.0 & 0.27 & 0.26 & 0.27 & 0.22 & 0.23 & 0.21 \\
\hline & 7.5 & 0.27 & 0.31 & 0.30 & 0.32 & 0.35 & 0.29 \\
\hline & 10.0 & 0.34 & 0.33 & 0.35 & 0.37 & 0.37 & 0.37 \\
\hline \multirow{5}{*}{0.05} & 0.5 & 0.00 & 0.00 & 0.00 & 0.00 & 0.00 & 0.00 \\
\hline & 2.5 & 1.17 & 1.08 & 1.33 & 0.93 & 0.65 & 1.35 \\
\hline & 5.0 & 1.00 & 1.15 & 0.77 & 1.41 & 1.47 & 1.27 \\
\hline & 7.5 & 2.32 & 1.13 & 1.24 & 2.09 & 2.12 & 1.88 \\
\hline & 10.0 & 2.96 & 2.60 & 2.11 & 2.79 & 2.46 & 2.56 \\
\hline \multirow{5}{*}{0.10} & 0.5 & 0.00 & - & - & 0.00 & 0.00 & 0.00 \\
\hline & 2.5 & 1.63 & 2.06 & 0.00 & 0.51 & 1.90 & 0.97 \\
\hline & 5.0 & 1.59 & 0.59 & 0.85 & 2.14 & 1.59 & 1.66 \\
\hline & 7.5 & 3.93 & 2.17 & 0.45 & 3.25 & 1.95 & 2.71 \\
\hline & 10.0 & 4.56 & 2.80 & 1.70 & 4.88 & 3.71 & 5.09 \\
\hline
\end{tabular}

Table 15

Relative profit improvement, $\left(\Delta \kappa^{*}\right)$, of dynamic pricing over Heuristic IV $\left(r=0.01, c_{o}=0.3 / a, C=10\right)$.

\begin{tabular}{|c|c|c|c|c|c|c|c|}
\hline \multirow[t]{2}{*}{$a$} & \multirow[t]{2}{*}{$\tau$} & \multicolumn{3}{|l|}{$b=3$} & \multicolumn{3}{|l|}{$b=4$} \\
\hline & & $\pi=5$ & $\pi=10$ & $\pi=20$ & $\pi=5$ & $\pi=10$ & $\pi=20$ \\
\hline \multirow{5}{*}{0.01} & 0.5 & 0.00 & 0.00 & 0.00 & 0.00 & 0.00 & 0.00 \\
\hline & 2.5 & 0.04 & 0.05 & 0.04 & 0.11 & 0.17 & 0.22 \\
\hline & 5.0 & 0.26 & 0.29 & 0.34 & 0.38 & 0.42 & 0.46 \\
\hline & 7.5 & 0.45 & 0.46 & 0.43 & 0.50 & 0.53 & 0.57 \\
\hline & 10.0 & 0.50 & 0.51 & 0.54 & 0.61 & 0.64 & 0.65 \\
\hline \multirow{5}{*}{0.05} & 0.5 & 0.00 & 0.00 & 0.00 & 0.00 & 0.00 & 0.00 \\
\hline & 2.5 & 0.00 & 0.00 & 0.00 & 0.00 & 0.00 & 0.00 \\
\hline & 5.0 & 0.00 & 0.00 & 0.00 & 0.05 & 0.07 & 0.15 \\
\hline & 7.5 & 0.01 & 0.00 & 0.02 & 0.26 & 0.33 & 0.28 \\
\hline & 10.0 & 0.05 & 0.21 & 0.07 & 0.55 & 0.45 & 0.48 \\
\hline \multirow{5}{*}{0.10} & 0.5 & 0.00 & - & - & 0.00 & 0.00 & 0.00 \\
\hline & 2.5 & 0.00 & 0.00 & 0.00 & 0.00 & 0.00 & 0.00 \\
\hline & 5.0 & 0.00 & 0.00 & 0.00 & 0.00 & 0.00 & 0.00 \\
\hline & 7.5 & 0.00 & 0.00 & 0.00 & 0.01 & 0.08 & 0.03 \\
\hline & 10.0 & 0.00 & 0.00 & 0.00 & 0.08 & 0.04 & 0.13 \\
\hline
\end{tabular}

Table 16

Relative profit improvement, $\left(\Delta \kappa^{*}\right)$, of dynamic pricing over Heuristic I $\left(r=0.1, c_{o}=0.3 / a, C=10\right)$.

\begin{tabular}{|c|c|c|c|c|c|c|c|}
\hline \multirow[t]{2}{*}{$a$} & \multirow[t]{2}{*}{$\tau$} & \multicolumn{3}{|l|}{$b=3$} & \multicolumn{3}{|l|}{$b=4$} \\
\hline & & $\pi=5$ & $\pi=10$ & $\pi=20$ & $\pi=5$ & $\pi=10$ & $\pi=20$ \\
\hline \multirow{7}{*}{0.01} & 0.5 & 0.00 & 0.00 & 0.00 & 0.34 & 0.36 & 0.42 \\
\hline & 2.5 & 0.43 & 0.46 & 0.51 & 1.32 & 1.37 & 1.47 \\
\hline & 5.0 & 1.26 & 1.25 & 1.21 & 2.42 & 2.48 & 2.59 \\
\hline & 7.5 & 1.62 & 1.66 & 1.76 & 3.20 & 3.26 & 3.37 \\
\hline & 10.0 & 2.11 & 2.15 & 2.12 & 3.95 & 3.97 & 4.00 \\
\hline & 0.5 & 0.00 & 0.00 & 0.00 & 0.00 & 0.00 & 0.00 \\
\hline & 2.5 & 0.44 & 0.25 & 0.25 & 1.04 & 0.84 & 0.97 \\
\hline
\end{tabular}


Table 16 (continued)

\begin{tabular}{|c|c|c|c|c|c|c|c|}
\hline \multirow[t]{2}{*}{$a$} & \multirow[t]{2}{*}{$\tau$} & \multicolumn{3}{|l|}{$b=3$} & \multicolumn{3}{|l|}{$b=4$} \\
\hline & & $\pi=5$ & $\pi=10$ & $\pi=20$ & $\pi=5$ & $\pi=10$ & $\pi=20$ \\
\hline \multirow[t]{5}{*}{0.05} & 5.0 & 0.49 & 0.52 & 0.57 & 1.77 & 1.52 & 1.78 \\
\hline & 7.5 & 0.70 & 0.64 & 0.77 & 2.34 & 2.48 & 2.34 \\
\hline & 10.0 & 0.80 & 0.85 & 0.90 & 2.96 & 2.97 & 3.00 \\
\hline & 0.5 & 0.00 & - & - & 0.00 & 0.00 & 0.00 \\
\hline & 2.5 & 0.13 & 0.05 & 0.00 & 0.88 & 0.57 & 0.61 \\
\hline \multirow[t]{3}{*}{0.10} & 5.0 & 0.40 & 0.24 & 0.47 & 1.18 & 1.16 & 1.09 \\
\hline & 7.5 & 0.56 & 0.59 & 0.36 & 1.50 & 1.67 & 1.43 \\
\hline & 10.0 & 0.46 & 0.37 & 0.66 & 1.78 & 1.80 & 1.75 \\
\hline
\end{tabular}

Table 17

Relative profit improvement, ( $\left.\Delta \kappa^{*}\right)$, of dynamic pricing over Heuristic II $\left(r=0.1, c_{o}=0.3 / a, C=10\right)$.

\begin{tabular}{|c|c|c|c|c|c|c|c|}
\hline \multirow[t]{2}{*}{$a$} & \multirow[t]{2}{*}{$\tau$} & \multicolumn{3}{|l|}{$b=3$} & \multicolumn{3}{|l|}{$b=4$} \\
\hline & & $\pi=5$ & $\pi=10$ & $\pi=20$ & $\pi=5$ & $\pi=10$ & $\pi=20$ \\
\hline \multirow{5}{*}{0.01} & 0.5 & 0.00 & 0.00 & 0.00 & 2.41 & 2.30 & 2.09 \\
\hline & 2.5 & 16.48 & 15.52 & 13.95 & 23.83 & 22.93 & 21.60 \\
\hline & 5.0 & 28.74 & 27.86 & 26.49 & 36.28 & 35.64 & 34.74 \\
\hline & 7.5 & 33.85 & 32.99 & 32.09 & 41.73 & 41.59 & 40.75 \\
\hline & 10.0 & 35.40 & 35.37 & 34.37 & 43.47 & 43.39 & 43.22 \\
\hline \multirow{5}{*}{0.05} & 0.5 & 0.00 & 0.00 & 0.00 & 0.00 & 0.00 & 0.00 \\
\hline & 2.5 & 11.22 & 9.26 & 8.95 & 19.57 & 18.47 & 16.15 \\
\hline & 5.0 & 20.61 & 19.62 & 17.10 & 30.31 & 29.96 & 28.99 \\
\hline & 7.5 & 20.31 & 21.21 & 19.99 & 31.43 & 31.90 & 30.51 \\
\hline & 10.0 & 17.06 & 17.74 & 19.64 & 26.38 & 27.14 & 27.88 \\
\hline \multirow{5}{*}{0.10} & 0.5 & 0.00 & - & - & 0.00 & 0.00 & 0.00 \\
\hline & 2.5 & 7.77 & 7.24 & 2.61 & 13.28 & 10.77 & 11.00 \\
\hline & 5.0 & 11.45 & 9.54 & 13.02 & 20.74 & 20.78 & 22.45 \\
\hline & 7.5 & 6.96 & 8.39 & 11.74 & 14.55 & 16.66 & 19.37 \\
\hline & 10.0 & 2.47 & 3.22 & 5.27 & 9.74 & 11.18 & 13.13 \\
\hline
\end{tabular}

Table 18

Relative profit improvement, $\left(\Delta \kappa^{*}\right)$, of dynamic pricing over Heuristic III $\left(r=0.1, c_{o}=0.3 / a, C=10\right)$.

\begin{tabular}{|c|c|c|c|c|c|c|c|}
\hline \multirow[t]{2}{*}{$a$} & \multirow[t]{2}{*}{$\tau$} & \multicolumn{3}{|l|}{$b=3$} & \multicolumn{3}{|l|}{$b=4$} \\
\hline & & $\pi=5$ & $\pi=10$ & $\pi=20$ & $\pi=5$ & $\pi=10$ & $\pi=20$ \\
\hline \multirow{5}{*}{0.01} & 0.5 & 0.00 & 0.00 & 0.00 & 0.12 & 0.18 & 0.22 \\
\hline & 2.5 & 0.36 & 0.34 & 0.30 & 0.22 & 0.20 & 0.20 \\
\hline & 5.0 & 0.31 & 0.30 & 0.39 & 0.29 & 0.28 & 0.28 \\
\hline & 7.5 & 0.45 & 0.48 & 0.47 & 0.41 & 0.37 & 0.39 \\
\hline & 10.0 & 0.63 & 0.57 & 0.59 & 0.51 & 0.53 & 0.47 \\
\hline \multirow{5}{*}{0.05} & 0.5 & 0.00 & 0.00 & 0.00 & 0.00 & 0.00 & 0.00 \\
\hline & 2.5 & 1.09 & 0.93 & 0.45 & 0.85 & 0.59 & 0.70 \\
\hline & 5.0 & 1.31 & 1.81 & 0.64 & 1.04 & 1.08 & 1.04 \\
\hline & 7.5 & 1.95 & 1.75 & 2.44 & 2.26 & 1.98 & 1.82 \\
\hline & 10.0 & 2.72 & 2.37 & 2.33 & 2.84 & 2.61 & 2.47 \\
\hline \multirow{5}{*}{0.10} & 0.5 & 0.00 & - & - & 0.00 & 0.00 & 0.00 \\
\hline & 2.5 & 1.52 & 0.05 & 0.00 & 1.81 & 0.85 & 1.06 \\
\hline & 5.0 & 1.21 & 2.59 & 0.87 & 2.40 & 1.34 & 0.61 \\
\hline & 7.5 & 3.84 & 4.00 & 1.97 & 3.20 & 2.57 & 1.48 \\
\hline & 10.0 & 2.75 & 5.08 & 4.31 & 4.07 & 3.56 & 3.52 \\
\hline
\end{tabular}


Table 19

Relative profit improvement, $\left(\Delta \kappa^{*}\right)$, of dynamic pricing over Heuristic IV $\left(r=0.1, c_{o}=0.3 / a, C=10\right)$.

\begin{tabular}{|c|c|c|c|c|c|c|c|}
\hline \multirow[t]{2}{*}{$a$} & \multirow[t]{2}{*}{$\tau$} & \multicolumn{3}{|l|}{$b=3$} & \multicolumn{3}{|l|}{$b=4$} \\
\hline & & $\pi=5$ & $\pi=10$ & $\pi=20$ & $\pi=5$ & $\pi=10$ & $\pi=20$ \\
\hline \multirow{5}{*}{0.01} & 0.5 & 0.00 & 0.00 & 0.00 & 0.00 & 0.00 & 0.00 \\
\hline & 2.5 & 0.00 & 0.01 & 0.04 & 0.14 & 0.12 & 0.16 \\
\hline & 5.0 & 0.23 & 0.25 & 0.28 & 0.38 & 0.40 & 0.43 \\
\hline & 7.5 & 0.43 & 0.42 & 0.43 & 0.57 & 0.57 & 0.59 \\
\hline & 10.0 & 0.57 & 0.59 & 0.60 & 0.75 & 0.74 & 0.75 \\
\hline \multirow{5}{*}{0.05} & 0.5 & 0.00 & 0.00 & 0.00 & 0.00 & 0.00 & 0.00 \\
\hline & 2.5 & 0.00 & 0.00 & 0.00 & 0.00 & 0.00 & 0.00 \\
\hline & 5.0 & 0.00 & 0.00 & 0.00 & 0.03 & 0.02 & 0.11 \\
\hline & 7.5 & 0.00 & 0.00 & 0.00 & 0.12 & 0.23 & 0.13 \\
\hline & 10.0 & 0.00 & 0.00 & 0.02 & 0.35 & 0.31 & 0.33 \\
\hline \multirow{5}{*}{0.10} & 0.5 & 0.00 & - & - & 0.00 & 0.00 & 0.00 \\
\hline & 2.5 & 0.00 & 0.00 & 0.00 & 0.00 & 0.00 & 0.00 \\
\hline & 5.0 & 0.00 & 0.00 & 0.00 & 0.00 & 0.00 & 0.00 \\
\hline & 7.5 & 0.00 & 0.00 & 0.00 & 0.00 & 0.00 & 0.00 \\
\hline & 10.0 & 0.00 & 0.00 & 0.00 & 0.00 & 0.00 & 0.00 \\
\hline
\end{tabular}

then decreasing in $\pi$ and $\tau$. The overall behavior can be explained through the impact of perishing; as it lessens, the myopic heuristic starts performing well. Incorporating future sales' contribution significantly improves the performance of the heuristics.

The single-price policy, Heuristic I, performs better than the myopic policy. Its performance with respect to the optimal improves with increasing menu cost, price sensitivity and discount rate, and with decreasing base demand rate and unit perishing cost. Only, in a few cases, when the shelf life is very small, both policies yield the same optimal discounted expected profit. Across all instances tested, the relative improvement achieved by dynamic pricing over Heuristic I is found to vary between $0 \%$ and $5.57 \%$ for a discount rate of $r=0.1$, whereas, it is between $0 \%$ and $7.52 \%$ for a smaller discount rate of $r=0.01$. This finding is interesting in that Zhao and Zheng (2000) also observed a similar range of improvement for the case of continuous pricing with zero price change costs. Thus, our results support their finding in the setting where pricing decisions are restricted to demand arrival instances.

Heuristic III dominates Heuristic I significantly for high price sensitivity and for low base demand rate and unit perishing cost. This behavior is confounded as the discount rate increases. Its overall performance with respect to the optimal worsens as $\tau$ increases.

Heuristic IV has the best performance among the tested heuristics. With low discount rates, it gives the optimal solution in most cases. As expected, its performance with respect to the optimal improves with increasing menu cost. Although its behavior is not monotone, we can make the following general observation: heuristic IV's performance deteriorates somewhat with increasing shelf life, unit perishing cost and base demand rate; it improves with higher price sensitivity. The relatively poor performance for small $a$ can be explained through the lessened impact of a pricing decision made early in the horizon on the revenues generated later. Heuristic IV performs surprisingly well given its simple construction. It is also amenable to exact computation of the optimal price; and, hence, is easily implementable.

\section{Conclusion}

In this study, we consider dynamic pricing of perishable items in an inventory system with a fixed shelf life and price-dependent stochastic demand. Price is allowed to change in both directions, i.e. both markup or markdown is possible at any demand point. As a novel feature, in our model, we assume that there are positive fixed price change costs. Revenues are collected and costs are incurred as the items are sold. Costs involved are the holding and perishing costs. All the cash flows are discounted at a discount rate $r$. We assume that unsold items have negligible salvage value, purchasing and ordering costs are sunk costs. The objective is to maximize the discounted expected profit by determining the selling prices dynamically. The pricing decisions are given at the instances at which demands occur and inventory is depleted. We formulate the problem as a DP problem and also consider joint determination of initial stock as well as optimal pricing.

We obtain some structural properties of the objective function with respect to the selling price and the horizon length, which are of theoretical interest and of practical use in the numerical solution of the DP formulations.

In a numerical study, we examine the sensitivity of the price decisions to various system parameters and compare the benefits of dynamic pricing policies versus four implementable policy heuristics. A single-period-look-ahead heuristic resulted in the worst performance. Incorporation of future sales' contribution significantly improves the performance of a heuristic. The fixed price heuristic is found to result in deviations between $0 \%$ and $5.57 \%$ for a discount 
rate of $r=0.1$, and between $0 \%$ and $7.52 \%$ for a smaller discount rate of $r=0.01$. A modified single price policy which uses the optimal fixed price computed dynamically for the remaining periods of the horizon after each demand occurrence performs extremely well. Across all instances tested, its deviation was never above $1 \%$, and it resulted in the optimal solution in most cases.

A number of extensions of our work are possible. The introduction of learning and updating of demand process over time may be an interesting and worthwhile extension. Another important research area for future studies may include different demand rate structures other than the linear case employed in our numerical study. A demand rate which is a function of both the remaining shelf life and the current price may be included in the setting we proposed.

\section{References}

Aggarwal, S.P., Jaggi, C.K., 1989. Ordering policy for decaying inventory. International Journal of Systems Science 20,151-155.

Blanchard, O.J., Fischer, S., 1989. Lectures on Macroeconomics. MIT Press, Cambridge, Massachusetts.

Cohen, M.A., 1977. Joint pricing and ordering policy for exponentially decaying inventory with known demand. Naval Research Logistics Quarterly 24, 257-268.

Diai, Y., Chiao, X., Fang, S., Nuttle, H.L.W., 2005. Pricing in revenue management for multiple firms competing for customers. International Journal of Production Economics 98, 1-16.

Eilon, S., Mallya, R.V., 1966. Issuing and pricing policies for semiperishables. In: Proceedings of Fourth International Conference on Operational Research. Wiley-Intersciences.

Federgruen, A., Heching, A., 1999. Combined pricing and inventory control under uncertainty. Operations Research 47, 454-475.
Feng, Y., Gallego, G., 1995. Optimal stopping times for end of season sales and optimal stopping times for promotional fares. Management Science 41, 1371-1391.

Feng, Y., Gallego, G., 1996. Perishable asset revenue management with Markovian time dependent demand intensities. Technical Report, Department of Industrial Engineering and Operational Research, Columbia University, New York.

Feng, Y., Xiao, B., 1999. Maximizing revenue of perishable assets with a risk factor. Operations Research 47, 337-341.

Feng, Y., Xiao, B., 2000a. A continuous time yield management model with multiple prices and reversible price changes. Management Science 46, 644-657.

Feng, Y., Xiao, B., 2000b. Optimal policies of yield management with multiple predetermined prices. Operations Research 48, 332-343.

Gallego, G., van Ryzin, G., 1994. Optimal dynamic pricing of inventories with stochastic demand over finite horizons. Management Science 40, 999-1020.

Kang, S., Kim, I., 1983. A study on the price and production level of the deteriorating inventory system. International Journal of Production Research 21, 440-460.

Maglaras, C., Meissner, J., 2006. Dynamic pricing strategies for multiproduct revenue management problems. Manufacturing \& Service Operations Management 8, 136-148.

Netessine, S., 2006. Dynamic pricing of inventory/capacity with infrequent price changes. European Journal of Operational Research 174, 533-580.

Rajan, A., Steinberg, R., Steinberg, R., 1992. Dynamic pricing and ordering decisions by a monopolist. Management Science 38, 240-262.

Subrahmanyan, S., Shoemaker, R., 1996. Developing optimal pricing and inventory policies for retailers who face uncertain demand. Journal of Retailing 72, 7-30.

Talluri, K.T., van Ryzin, G.J., 2004. The Theory and Practice of Revenue Management. Springer, New York.

Yildirim, G., 2001. A dynamic pricing policy for perishables with stochastic demand. Master's Thesis, Bilkent University, Ankara, Turkey.

Zhao, W., Zheng, Y.-S., 2000. Optimal dynamic pricing for perishable assets with nonhomogeneous demand. Management Science 46, 375-388. 